\title{
Vortex breakdown in a truncated conical bioreactor
}

Balci, Adnan; Brøns, Morten; Herrada, Miguel A. ; Shtern, Vladimir N.

Published in:

Fluid Dynamics Research

Link to article, DOI:

10.1088/0169-5983/47/6/065503

Publication date:

2015

Document Version

Publisher's PDF, also known as Version of record

Link back to DTU Orbit

Citation (APA):

Balci, A., Brøns, M., Herrada, M. A., \& Shtern, V. N. (2015). Vortex breakdown in a truncated conical bioreactor. Fluid Dynamics Research, 47(6), [065503]. https://doi.org/10.1088/0169-5983/47/6/065503

\section{General rights}

Copyright and moral rights for the publications made accessible in the public portal are retained by the authors and/or other copyright owners and it is a condition of accessing publications that users recognise and abide by the legal requirements associated with these rights.

- Users may download and print one copy of any publication from the public portal for the purpose of private study or research.

- You may not further distribute the material or use it for any profit-making activity or commercial gain

- You may freely distribute the URL identifying the publication in the public portal

If you believe that this document breaches copyright please contact us providing details, and we will remove access to the work immediately and investigate your claim. 
Vortex breakdown in a truncated conical bioreactor

This content has been downloaded from IOPscience. Please scroll down to see the full text. 2015 Fluid Dyn. Res. 47065503

(http://iopscience.iop.org/1873-7005/47/6/065503)

View the table of contents for this issue, or go to the journal homepage for more

Download details:

This content was downloaded by: makalakat

IP Address: 130.225.69.179

This content was downloaded on 06/10/2015 at 08:31

Please note that terms and conditions apply. 


\title{
Vortex breakdown in a truncated conical bioreactor
}

\author{
Adnan Balci ${ }^{1}$, Morten Brøns ${ }^{1,4}$, Miguel A Herrada ${ }^{2}$ and \\ Vladimir N Shtern ${ }^{3}$
}

${ }^{1}$ DTU Compute, Technical University of Denmark, DK-2800 Kgs. Lyngby, Denmark ${ }^{2}$ E.S.I, Universidad de Sevilla, Camino de los Descubrimientos s/n, E-41092, Spain

${ }^{3}$ Shtern Research and Consulting, Houston, TX 77096, USA

E-mail: mobr@dtu.dk

Received 7 June 2015, revised 28 July 2015

Accepted for publication 1 September 2015

Published 1 October 2015

Communicated by Andrew David Gilbert

\begin{abstract}
This numerical study explains the eddy formation and disappearance in a slow steady axisymmetric air-water flow in a vertical truncated conical container, driven by the rotating top disk. Numerous topological metamorphoses occur as the water height, $H_{\mathrm{w}}$, and the bottom-sidewall angle, $\alpha$, vary. It is found that the sidewall convergence (divergence) from the top to the bottom stimulates (suppresses) the development of vortex breakdown (VB) in both water and air. At $\alpha=60^{\circ}$, the flow topology changes eighteen times as $H_{\mathrm{w}}$ varies. The changes are due to (a) competing effects of AMF (the air meridional flow) and swirl, which drive meridional motions of opposite directions in water, and (b) feedback of water flow on AMF. For small $H_{\mathrm{w}}$, the AMF effect dominates. As $H_{\mathrm{w}}$ increases, the swirl effect dominates and causes VB. The water flow feedback produces and modifies air eddies. The results are of fundamental interest and can be relevant for aerial bioreactors.
\end{abstract}

Keywords: vortex breakdown, flow topology, Moffatt eddies, bioreactors, axisymmetric flow

(Some figures may appear in colour only in the online journal)

${ }^{4}$ Author to whom any correspondence should be addressed. 


\section{Introduction}

The conical geometry is beneficial for vortex devices. Examples are hydrocyclones (Schultz et al 2009) and vortex tubes (Secchiaroli et al 2009). Their large length-to-radius ratio causes that swirl significantly decays downstream due to friction at the sidewall. Since the angular momentum, $r v$, is nearly conserved in a fast flow, where $v$ is the swirl velocity and $r$ is the distance from the rotation axis, the reduction of $r$ in a conical part of a vortex device results in increasing $v$ that helps sustain the strong centrifugal force.

This effect of conical geometry can also be useful for bioreactors. Aerial vortex bioreactors are a rapidly developing technology. They employ air-water flows for the efficient growth of tissue culture. A rotating disk (Liow et al 2009) or a propeller (Ramazanov et al 2007), located in the air part of flow, induces a swirling motion. The swirling air converges toward the reactor axis near the interface and drives a slow rotation and the meridional circulation of water. The air meridional circulation delivers to the interface oxygen required for the tissue culture growth. The water circulation enhances mixing of the dissolved oxygen with other ingredients. The tissue growth is a time-consuming process requiring a slow motion of ingredients and small shear stresses. The air driving satisfies these requirements.

The commercial aerial bioreactors are cylindrical (Ramazanov et al 2007). The study by Herrada and Shtern (2014) revealed a non-trivial topology of a creeping air-water flow in a vertical sealed cylinder driven by the rotating top disk. As the volume fraction of water increases, eddies develop and multiple topological changes occur in both air and water motions despite the fact that the flow being extremely slow. Such paradoxical complexity of a creeping flow is caused by competing effects of the swirl and meridional motions. This makes the fluid mechanics of aerial bioreactors of fundamental interest.

The swirl rapidly decays from the rotating top toward the bottom of aerial bioreactor due to friction at walls and viscous diffusion dominating in a slow motion. The conical geometry can at least partially compensate the swirl decay. This potentially beneficial effect is a practical motivation of our study.

Another motivation is fundamental: the vortex breakdown (VB) physics. The conical geometry strongly affects the development of local circulation regions in swirling flows which are often referred to as vortex breakdown bubbles (VBBs). To better understand the VB nature, numerous studies have been performed since 1957 , motivated by the VB relevance for delta-wing aircraft, vortex combustors and tornados (Escudier 1988, Shtern 2012a). Among many VB explanations, Benjamin's theory (1962), interpreting VB as an inertial wave roll-up, seems to be the most popular, having more than 550 citations, but unfortunately the theory is not consistent with the observed important VB features (Shtern and Hussain 1999). The authors follow the definition by Leibovich (1978) that VB is a reversal of the axial velocity. For the reversal, only the swirl-to-meridional-motion strength ratio is important and therefore VB can occur in a slow motion as well (Herrada and Shtern 2014).

The breakthrough in the understanding of the VB nature was achieved due to fundamental research of swirling flows in sealed containers and, e.g., by Lopez (1990), Valentine and Jahnke (1994) and Mullin et al (2000). In particular, it was shown that the container conical shape can help suppress the near-axis VB while it can induce a local circulation region near the sidewall (Escudier et al 2007). These effects of conical geometry are a motivation for our study which generalizes the results of Escudier et al (2007) to air-water flows.

In contrast to the one-fluid case, VB occurs even in a creeping air-water motion. There the water fraction, $H_{\mathrm{w}}$, replaces the Reynolds number, $R e$, as a key parameter, controlling the flow 


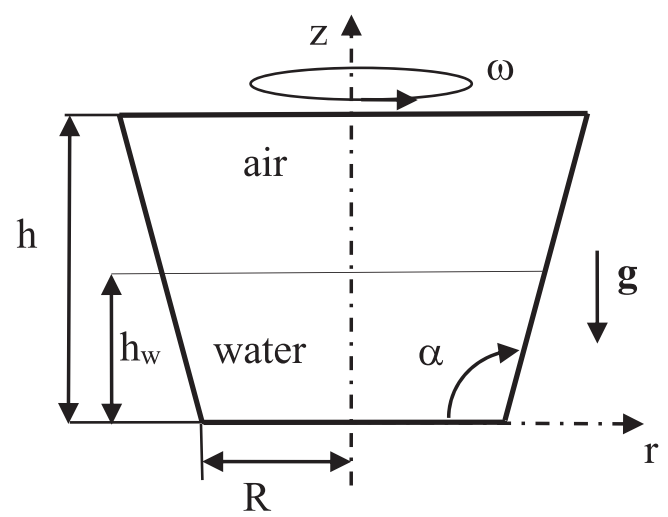

Figure 1. Schematic of the problem.

topology. For small $H_{\mathrm{w}}$, the air meridional motion effect dominates the swirl effect in water. With increasing $H_{\mathrm{w}}$, the water rotation speeds up and causes VBs first into water and then in air.

The nature of the VB development in water is the same as that in the single-fluid flows where VB occurs via the swirl decay mechanism (Herrada et al 2015). The results described in section 7 reveal that as $R e$ increases, the VB in air disappears, but the VB in water does not. The water VBB in the creeping flow eventually transforms into the water VBB in the high-Re flow (Herrada et al 2013).

The feature that VB and other topological metamorphoses occur in a very slow flow is counterintuitive. It might be superficially believed that a creeping flow, dominated by viscous diffusion, should have a simple topology. However, the Moffatt (1964) discovery of an infinite set of eddies in a creeping flow between two inclined walls destroyed this illusion.

Numerous studies, following the Moffatt finding, revealed that the cellular creeping motions are wide-spread, occurring in a plane cavity (Moffatt 1964, Shankar and Deshpande 2000), cone (Wakiya 1976), cylinder (Blake 1979, Hills 2001), in cavities with oppositely moving walls (Gurcan et al 2003, Wilson et al 2005), between concentric cones (Hall et al 2007) and coaxial cylinders (Shtern 2012b).

The topology of two-fluid flows is more complicated than that of one-fluid flows. Herrada and Shtern (2014) revealed nine topological changes in the creeping air-water flow in a sealed cylinder as the water volume fraction, $H_{\mathrm{w}}$, increases. Our paper demonstrates that this number is indeed larger for air-water flows in both cylindrical and conical containers and focuses on the effects of conical geometry.

In the rest of this paper, we formulate the problem (section 2), describe the numerical technique (section 3), explore the flow pattern for small $H_{\mathrm{w}}$ (section 4), investigate how the pattern changes as $H_{\mathrm{w}}$ grows in cones converging (section 5) and diverging (section 6) from the rotating lid to the stationary bottom disk, study the effect of increasing rotation (section 7), summarize the results in section 8 .

\section{Problem formulation}

\subsection{Flow geometry}

Figure 1 is a problem schematic. The lower part, $0<z<h_{\mathrm{w}}$, of the conical container is filled with water, the upper part, $h_{\mathrm{w}}<z<h$, is filled with air; $h$ is the truncated-cone height; $\alpha$ is 
the angle between the bottom and the sidewall; $\mathbf{g}$ is the gravitational acceleration. The interface is depicted by the thin horizontal line, $z=h_{\mathrm{w}}$. The top disk (at $z=h$ ) rotates with angular velocity $\omega$. The rotation strength can be characterized by the Reynolds number, $R e=\omega R^{2} / \nu_{\mathrm{w}} ; R$ is the bottom-disk radius, and $\nu_{\mathrm{w}}$ is the kinematic viscosity of water. The other control parameters are aspect ratio $H=h / R$ and the water volume fraction characterized by $H_{\mathrm{w}}=h_{\mathrm{w}} / h$. Since the aspect ratio of aerial bioreactors is typically close to one (Ramazanov et al 2007, Liow et al 2009), here $H=1$ is fixed while $H_{\mathrm{w}}$ varies.

Our study is limited to the case where the wall material does not change the angle between the interface and wall. The obtained results can be useful as a reference for further studies of the wall material effects. In a creeping flow the interface is undisturbed. Herrada et al (2013) and Herrada and Shtern (2014) found that the interface deformation also is negligibly small for moderate Re typical of bioreactor flows. No significant deformation of the interface was observed in the experiment of Lo Jacono et al (2009). The physical reason is the small air-to-water density ratio. Based on this reason and prior results, the interface is considered undisturbed in this study: $z=z_{\mathrm{i}}=H_{\mathrm{w}}$.

\subsection{Governing equations}

The governing equations are the same as in the paper by Herrada and Shtern (2014). They are rewritten below for convenience of the reader. Using $R, \omega R$, and $\rho_{\mathrm{w}} \omega^{2} R^{2}$ as scales for length, velocity, and pressure, respectively, renders all variables dimensionless. We consider steady axisymmetric flows of air and water both governed by the Navier-Stokes equations for a viscous incompressible fluid,

$$
\begin{aligned}
& r^{-1} \partial(r u) / \partial r+\partial w / \partial z=0 \\
& u \partial u / \partial r+w \partial u / \partial z=v^{2} / r-\rho_{n} \partial p / \partial r \\
& \quad+\nu_{n} \operatorname{Re}^{-1}\left(\nabla^{2} u-u / r^{2}\right) \\
& u \partial v / \partial r+w \partial v / \partial z+u v / r=\nu_{n} \operatorname{Re}^{-1}\left(\nabla^{2} v-v / r^{2}\right), \\
& u \partial w / \partial r+w \partial w / \partial z=-\rho_{n} \partial p / \partial z+\nu_{n} \operatorname{Re}^{-1}\left(\nabla^{2} w\right)
\end{aligned}
$$

where $\nabla^{2} \equiv r^{-1} \partial(r \partial / \partial r) / \partial r+\partial^{2} / \partial z^{2},(u, v, w)$ are the velocity components in the cylindrical coordinate, $(r, \phi, z)$, and $p$ is pressure reduced by the hydrostatic contribution. The coefficients, $\rho_{n}$ and $\nu_{n}$, both equal 1 for $n=1$ (in water) while $\rho_{n}=\rho_{\mathrm{w}} / \rho_{\mathrm{a}}$ and $\nu_{n}=\nu_{\mathrm{a}} / \nu_{\mathrm{w}}$ for $n=2$ (in air); $\nu_{\mathrm{w}}=10^{-6} \mathrm{~m}^{2} \mathrm{~s}^{-1}$ and $\nu_{\mathrm{a}}=15 \times 10^{-6} \mathrm{~m}^{2} \mathrm{~s}^{-1}$ are the kinematic viscosities; $\rho_{\mathrm{w}}=1000 \mathrm{~kg} \mathrm{~m}^{-3}$ and $\rho_{\mathrm{a}}=1.22 \mathrm{~kg} \mathrm{~m}^{-3}$ are the densities; subscripts 'a' and 'w' are abbreviations for 'air' and 'water'. This air density value corresponds to the atmospheric pressure and the room temperature.

\subsection{Boundary conditions}

Equations (1)-(4) are solved under the following boundary conditions:

(i) Regularity at the axis, $0<z<1, r=0$ : $u=v=0$, $\partial w / \partial r=0$.

(ii) No-slip at the walls: $u=v=w=0$ at the still disk, $0<r<1, z=0$, and at the sidewall, $0<z<1, r=1-z \cot \alpha ; u=w=0, v=r$ at the rotating disk, $0<r<1$ $-\cot \alpha, z=1$. 
(iii) Continuity of all the velocity and stress components at the air-water interface, $z=H_{\mathrm{w}}$. The normal-to-interface velocity is zero: $w=0$ at $z=H_{\mathrm{w}}$.

\subsection{Reduced problem}

As $R e \rightarrow 0$, the motion becomes very slow and the nonlinear terms become negligibly small compared with the linear terms in equation (3), which reduces to

$$
\nabla^{2} v-v / r^{2}=0
$$

This equation has a nonzero solution due to the boundary condition at the top disk: $v=r$ at $z=1$. At all other walls, the swirl velocity is zero, $v=0$. At the interface, $v$ and the swirl shear stresses are continuous: $v_{\mathrm{w}}=v_{\mathrm{a}}$ and $\partial v_{\mathrm{w}} / \partial z=\mu_{r} \partial v_{\mathrm{a}} / \partial z$ at $z=H_{\mathrm{w}}$. Thus the problem for the swirl velocity becomes separated from the problem for the meridional motion in the limiting case as $R e \rightarrow 0$, similar to that in the one-fluid problem studied by Hills (2001). We first solve this linear problem for swirl.

Next, we address the problem for the meridional motion. Since the boundary conditions are uniform, the meridional motion is only driven by the centrifugal force corresponding to term $v^{2} / r$ in equation (2). This term must be preserved, while the other nonlinear terms can be omitted in the limiting case as $R e \rightarrow 0$. Then introducing $u^{*}=u / \operatorname{Re}$ and $w^{*}=w / \operatorname{Re}$ reduces equations (1), (2), and (4) to

$$
\begin{aligned}
& r^{-1} \partial\left(r u^{*}\right) / \partial r+\partial w^{*} / \partial z=0, \\
& \rho_{n} \partial p / \partial r-v^{2} / r=\nu_{n}\left(\nabla^{2} u^{*}-u^{*} / r^{2}\right), \\
& \rho_{n} \partial p / \partial z=\nu_{n} \nabla^{2} w^{*} .
\end{aligned}
$$

It is interesting that the entire problem formally is nonlinear despite the fact that the motion being creeping, but can be divided into the two linear problems: one for the swirl velocity (5) and the other for the meridional motion (6)-(8). After solving problem (5), the 'source' term, $v^{2} / r$ in equation (7), is prescribed, so the problem for the meridional motion also is linear. This feature is also similar to that in the one-fluid flow studied by Hills (2001).

\section{Numerical procedure}

\subsection{Transformation of equations}

For the $R e$ ranges addressed in this work, the interface deformation is negligibly small and $r=r_{\mathrm{i}}=H_{\mathrm{w}}$ is a good approximation for the interface. The number of variables involved in the problem is reduced by introducing a stream-function-vorticity-angular-momentum form. System (1)-(4) is transformed into three equations for the Stokes stream function $\Psi$, $u=-r^{-1} \partial \Psi / \partial z, w=r^{-1} \partial \Psi / \partial r$, the azimuthal vorticity component, $\eta=\partial u / \partial z-\partial w / \partial r$, and circulation, $\Gamma=r v$ :

$$
\begin{aligned}
& \nabla^{2} \Psi-2 r^{-1} \partial \Psi / \partial r=-r \eta \\
& u \partial \eta / \partial r+w \partial \eta / \partial z-u \eta / r=2 r^{-3} \Gamma \partial \Gamma / \partial z \\
& \quad+\nu_{n} \operatorname{Re}^{-1}\left(\nabla^{2} \eta-\eta / r^{2}\right)
\end{aligned}
$$




$$
u \partial \Gamma / \partial r+w \partial \Gamma / \partial z=\nu_{n} \operatorname{Re}^{-1}\left(\nabla^{2} \Gamma-2 r^{-1} \partial \Gamma / \partial r\right)
$$

These equations are solved applying the boundary conditions for $\Psi, \eta$ and $\Gamma$ which follows from those listed in section 2.3.

\subsection{Linear problem}

In the limiting case as $R e \rightarrow 0$, introducing $\eta^{*}=\eta / R e$ and $\Psi^{*}=\Psi / R e$ reduces equations (9)-(11) to

$$
\begin{aligned}
& \nabla^{2} \Psi^{*}-2 r^{-1} \partial \Psi^{*} / \partial r=-r \eta^{*} \\
& 2 r^{-3} \Gamma \partial \Gamma / \partial z+\nu_{n}\left(\nabla^{2} \eta^{*}-\eta^{*} / r^{2}\right)=0 \\
& \nabla^{2} \Gamma-2 r^{-1} \partial \Gamma / \partial r=0
\end{aligned}
$$

\subsection{Discretization}

A boundary-fitted coordinate system is used to calculate the problem. Both the water and air regions are mapped onto the fixed rectangular domains (a) $0 \leqslant \varphi \leqslant 1,0 \leqslant \xi_{\mathrm{w}} \leqslant H_{\mathrm{w}}$, and (b) $0 \leqslant \varphi \leqslant 1, H_{\mathrm{w}} \leqslant \xi_{\mathrm{a}} \leqslant 1$. To this end, we perform the coordinate transformations: (a) $\varphi=r /(1-z \cot \alpha), \xi_{\mathrm{w}}=z$ and (b) $\varphi=r /(1-z \cot \alpha), \xi_{\mathrm{a}}=z$. These domains are discretized by using a set of $n_{\xi \mathrm{w}}$ and $n_{\xi \mathrm{a}}$ Chebychev spectral collocation points in the $\xi$ direction. The $\varphi$ interval is discretized using a set of $n_{\varphi}$ Chebychev spectral collocation points.

\subsection{Nonlinear problem}

The Newton iterative procedure is used to solve the discretized non-linear problem derived from (9)-(11) and their corresponding boundary conditions. Given an initial solution guess, a new approximate solution is found by solving the system of $3\left(n_{\xi \mathrm{w}}+n_{\xi \mathrm{a}}\right) \times n_{\varphi}$ linear algebraic equations for $\Psi, \eta$ and $\Gamma$ at the collocation points resulting from the Newton linearization. For a prescribed geometrical configuration, the simulation is started with a small value of the Reynolds number and the flow at rest. Once the approximate solution has converged, the converged solution is used as a new guess for a new run with a higher Reynolds number.

\subsection{Advantages of Chebyshev grid}

In the reduced problem, the discretized problem consists of (i) system of $\left(n_{\xi \mathrm{w}}+n_{\xi \mathrm{a}}\right) \times n_{\varphi}$ linear algebraic equations for $\Gamma$ and (ii) system of $2\left(n_{\xi \mathrm{w}}+n_{\xi \mathrm{a}}\right) \times n_{\varphi}$ linear algebraic equations for $\Psi^{*}$ and $\eta^{*}$ which are solved sequentially. All linear systems, in the full and reduced problems, are solved directly applying the standard procedure of matrix inversion.

For the presented results, the simulations are done mostly with $n_{\xi \mathrm{w}}=40, n_{\xi \mathrm{a}}=30$, and $n_{\varphi}=50$ (standard grid). In order to verify the grid independence, some runs have been carried out at $n_{\xi \mathrm{w}}=50, n_{\xi \mathrm{a}}=40$, and $n_{\varphi}=60$ (fine grid).

Since the Chebyshev grid points concentrate near the interface from both sides, the approach is adequate to resolve thin circulation layers, located near the interface, even using moderate values of $n_{\xi \mathrm{w}}$ and $n_{\xi \mathrm{a}}$. The Chebyshev grid points concentrate near the walls as well, that helps to better resolve the Moffatt and other corner eddies, even using a moderate value 

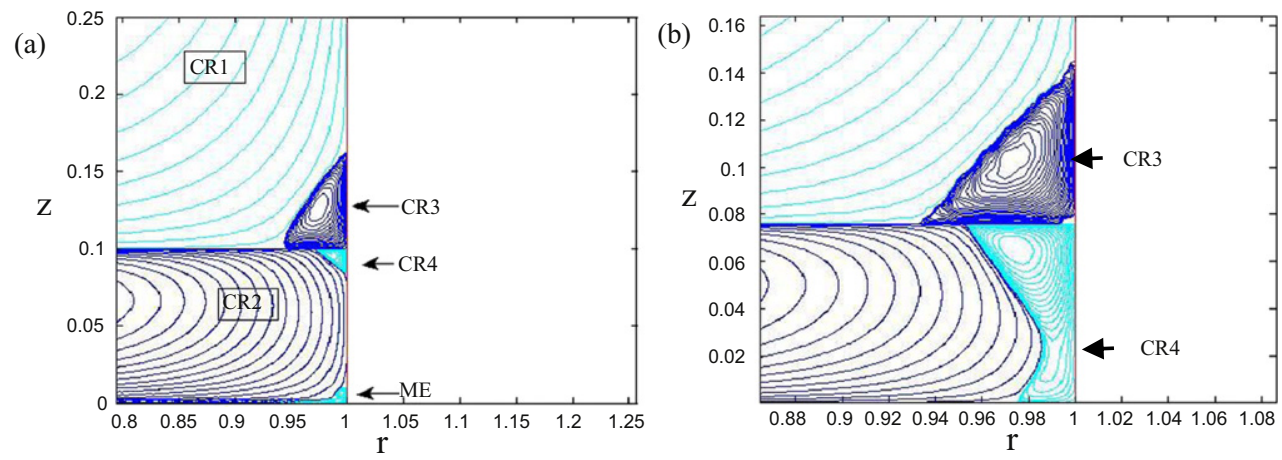

Figure 2. Merging of regions CR4 and ME as $H_{\mathrm{w}}$ decreases from 0.1 (a) to 0.072 (b).

(a)

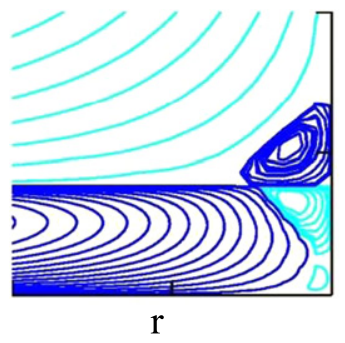

(b)

Z

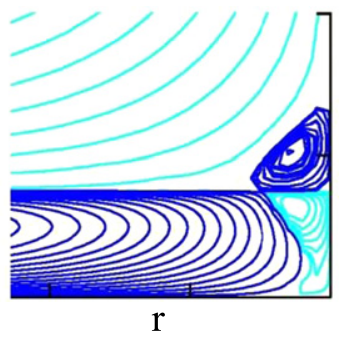

Figure 3. Details of merging depicted in figure 2: $H_{\mathrm{w}}=0.074$ (a) and 0.073 (b).

of $n_{\varphi}$. The Chebyshev grid points also concentrate near the axis that helps resolve small VBBs emerging near the axis-bottom and axis-interface intersections as $H_{\mathrm{w}}$ increases.

\section{Shallow water spout}

\subsection{Cylindrical geometry}

It is convenient to start with the cylindrical-container creeping flow and to discuss a topological transformation, as $H_{\mathrm{w}}$ decreases from 0.1 to zero, which was not described by Herrada and Shtern (2014). (Though we use the word 'decreases' and similar words, denoting a timedependent process, the paper only deals with steady flows established after changing a control parameter, e.g., $H_{\mathrm{w}}$.) Figure 2 depicts the streamline patterns of meridional flow near the bottom-sidewall intersection at $\alpha=90^{\circ}, H=1$ and (a) $H_{\mathrm{w}}=0.1$ and (b) $H_{\mathrm{w}}=0.072$. The results for $H_{\mathrm{w}}=0.1$ (figure 2(a)) coincide with those obtained by Herrada and Shtern (2014).

As $H_{\mathrm{w}}$ decreases, the water circulation region CR4 and the outmost Moffatt eddy (ME) shown in figure 2(a) merges into a circulation cell again marked as CR4 in figure 2(b). As $H_{\mathrm{w}}$ further decreases down to zero, no topological transformation occurs, just the water layer shrinks to the bottom.

Figure 3 depicts streamline patterns at $H_{\mathrm{w}}=0.074$ (a) and 0.073 (b) which are intermediate snapshots illustrating the merging of CR4 and ME. Figure 4 shows schematics explaining this topological transformation. The arrows in figure 4 indicate the flow directions. Figure 4(a) corresponds to figure 2(a) and emphasizes the saddle stagnation points of the water meridional motion, $S_{1}$ and $S_{2}$, and the center point $\mathrm{C}_{2}$ of the outmost ME. The smaller 

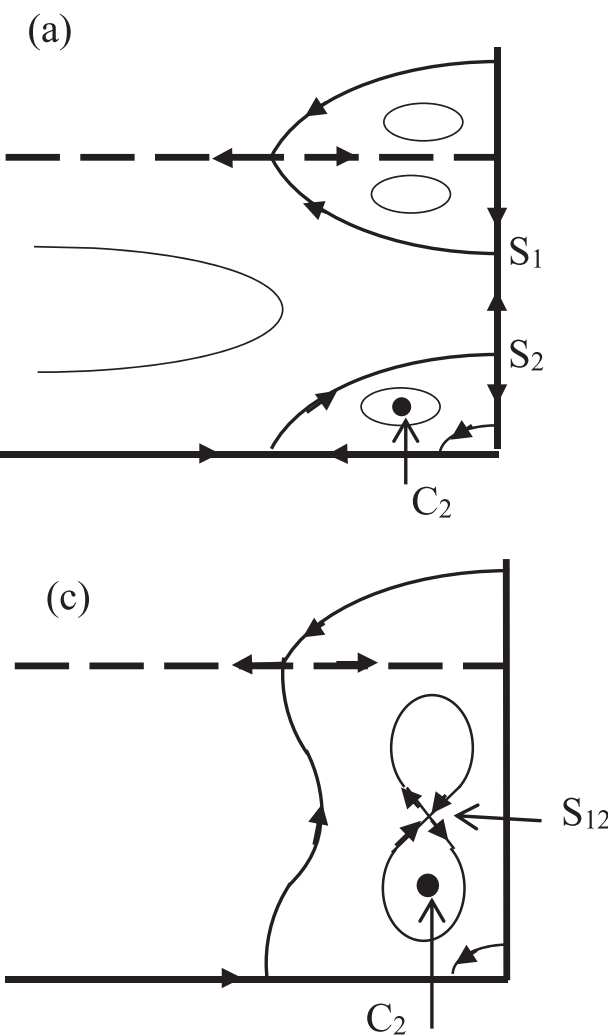

(b)
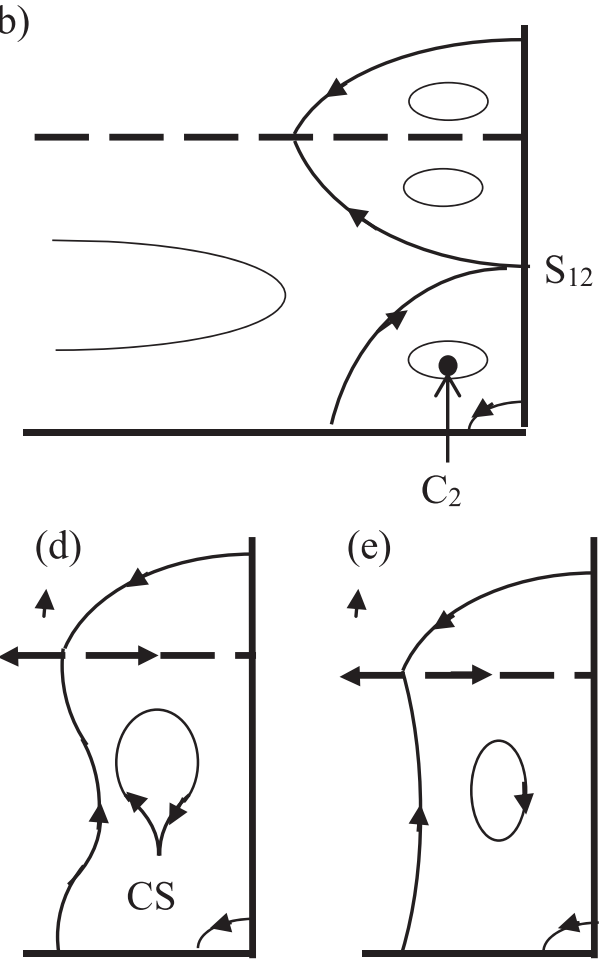

Figure 4. Schematics showing merging of saddle points $S_{1}$ and $S_{2}$ (a) into $S_{12}$ (b), separation of $S_{12}$ from sidewall (c) and merging of $S_{12}$ and center $\mathrm{C}_{2}$ (c) into cusp point CS (d) which then disappears (e).

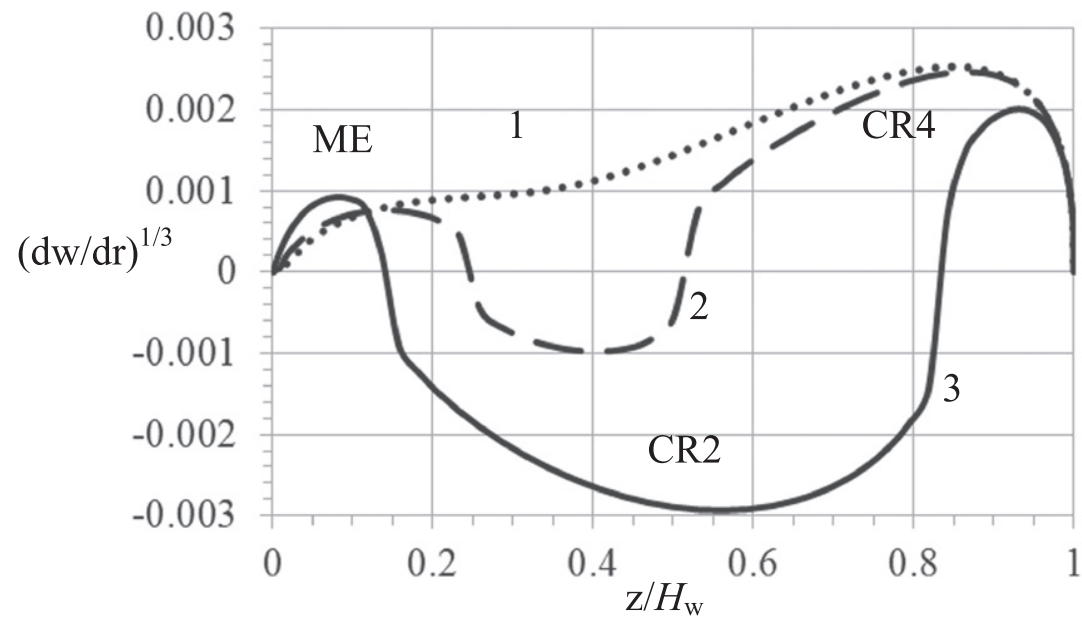

Figure 5. Profile of meridional shear stress at the sidewall in water at $H_{\mathrm{w}}=0.076$ (1), $0.08(2)$ and $0.1(3)$. 
(a)

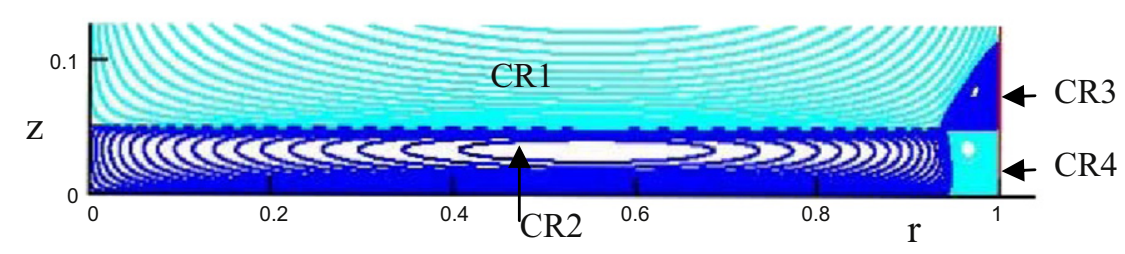

(b)

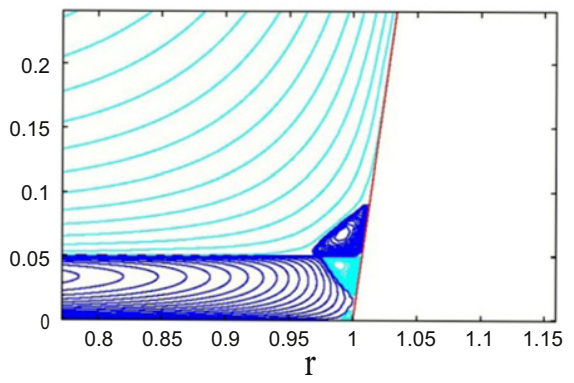

(c)

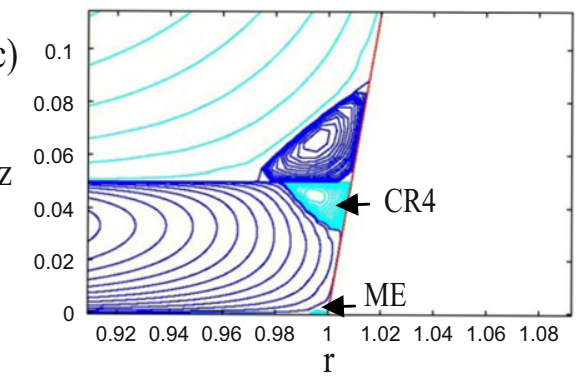

(d)

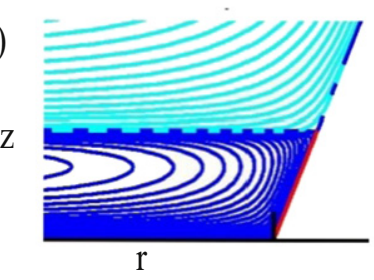

Figure 6. Transformation of near-interface eddies as cone angle increases: $\alpha=90^{\circ}$ (a), $98^{\circ}(\mathrm{b}), 100^{\circ}(\mathrm{c})$, and $120^{\circ}(\mathrm{d}) ; H_{\mathrm{w}}=0.05$.

ME is also depicted in figure 4 near the bottom-sidewall intersection. The smaller Moffatt vortices and all smaller vortices in regions CR3 and CR4 are not resolved by our numerical simulations. These vortices are not involved in bifurcations discussed in this paper.

As $H_{\mathrm{w}}$ decreases, $S_{1}$ and $S_{2}$ approach each other and merge into saddle point $S_{12}$ (figure 4(b)). Then $S_{12}$ separates from the sidewall, see the schematic in figure 4(c) which corresponds to the flow pattern in figure 3(a). As $H_{\mathrm{w}}$ further decreases, $S_{12}$ and center $\mathrm{C}_{2}$ merge into cusp point CS, see figure 4(d) which nearly corresponds to figure 3(b). Then these saddle points disappear and the streamline pattern becomes as shown in figures 4(e) and 2(b). This bifurcation was systematically studied by Brøns (2007). Thus, figure 2(b) represents the flow pattern, P1, which first develops as a small amount of water is added in the water-free container. Figure-eight streamline patterns, like shown in figure 4(c), appear (bifurcation A8) and disappear (bifurcation D8) in the topological scenarios, described below, where the notations, A8 and D8, are used for brevity.

Figure 5 depicts the dependence of the meridional stress, $\mathrm{d} w / \mathrm{d} r$, on $z$ at the sidewall, $r=1$, in water at $H_{\mathrm{w}}=0.076$ (curve 1), 0.08 (curve 2) and 0.1 (curve 3). The interval near $z=0$, where $\mathrm{d} w / \mathrm{d} r>0$, corresponds to the ME, the interval near $z=H_{\mathrm{w}}$, where $\mathrm{d} w /$ $\mathrm{d} r>0$, corresponds to the near-interface eddy CR4, and the in-between interval, where $\mathrm{d} w /$ $\mathrm{d} r<0$, corresponds to circulation region CR2 (figure 2(a)). Figure 5 illustrates how region CR2 touches the sidewall and expands at the sidewall as $H_{\mathrm{w}}$ increases. Interpolation yields that ME and CR4 meet at $H_{\mathrm{w}}=0.078$. 


\subsection{Conical geometry, $\alpha$ increases}

Now we explore how pattern P1 (figure 2(b)) varies with $\alpha$. Figure 6 depicts streamlines of the meridional motion at fixed $H_{\mathrm{w}}=0.05$ and $\alpha=90^{\circ}$ (a), $98^{\circ}$ (b), $100^{\circ}$ (c), and $120^{\circ}$ (d). As $\alpha$ varies from $90^{\circ}$ to $98^{\circ}$, circulation region CR2 expands up to the sidewall and region CR4 splits into the two parts: (i) the near-interface eddy, again denotes as CR4 in figure 6(c), and (ii) the ME (figure 6(c)) which, being very small, is not visible in figure 6(d). The character of this topological transformation, i.e., the transition from the pattern, shown in figure 6(c), to the pattern, shown in figure 6(a), is similar to that illustrated by figure 4 and discussed in section 4.1 .

The pattern, shown in figure $6(\mathrm{~d})$ for $\alpha=120^{\circ}$, is topologically identical with that at $\alpha=100^{\circ}$ (figure 6(c)), but the dimension of $\mathrm{ME}$ becomes too small to be visible in figure 6(d). Also the vertical extension of CR4 and the size of CR3 become too small to be visible in figure $6(\mathrm{~d})$.

However, these small regions do exist in this range of $\alpha$ as the $u>0$ ranges indicate in figure 7 , where the distribution of radial velocity, $u$, at the interface. $H_{\mathrm{w}}=0.05$, is plotted. We use $u^{1 / 5}$ in figure 7 in order to better observe the near-sidewall ranges of small positive $u$. Figure 7(a) depicts the $u$ distributions for $\alpha=90^{\circ}$ (curve 1) and $100^{\circ}$ (curve 2) at the entire interface, $0<r<r_{\text {is }}=1-H_{\mathrm{w}} \cot \alpha$, where $r_{\text {is }}$ is the radial coordinate of the interface-sidewall intersection. The $u<0$ intervals correspond to the boundary between regions CR1 and CR2 while the $u>0$ intervals correspond to the boundary between regions CR3 and CR4 (figure 6(a)). Figure 7(b) depicts the $u$ distributions for $\alpha=90^{\circ}$ (curve 1), $100^{\circ}$ (2), $110^{\circ}$ (3), $120^{\circ}(4)$, and $130^{\circ}(5)$ in the vicinity of the sidewall. Figure $7(b)$ reveals that the radial extent of CR3 and CR4 decreases as $\alpha$ increases, but remains nonzero even at $\alpha=130^{\circ}$. According to Moffatt (1964), ME should exist up to $\alpha=146^{\circ}$. Figure 7 agrees with the theory of Moffatt eddies near the interface-wall intersection (Shtern 2014). ME and CR4 merge at $H_{\mathrm{w}}=H_{\mathrm{w} 1}$, whose value decreases as $\alpha$ increases, e.g., $H_{\mathrm{w} 1}=0.078$ at $\alpha=90^{\circ}$ and $H_{\mathrm{w} 1}=0.05$ at $\alpha=98^{\circ}$.

\subsection{Conical geometry, $\alpha$ decreases}

In contrast to the case, where $\alpha$ increases and the near-sidewall eddies shrink (section 4.2), the eddies expand as $\alpha$ decreases. Moreover, a smaller near-interface eddy also becomes resolved by our standard grid, as it follows from figure 8 depicting the $u$ distributions at the interface for $\alpha=90^{\circ}$ (curve 1 ), $70^{\circ}$ (curve 2), and $60^{\circ}$ (curve 3). Figure 9 shows the streamline pattern near the bottom-sidewall intersection at $\alpha=70^{\circ}$ and $H_{\mathrm{w}}=0.05$.

\section{Topological metamorphoses of air-water flow in the $\alpha=120^{\circ}$ cone as $H_{\mathrm{w}}$ increases}

The first topological transformation, (i), as $H_{\mathrm{w}}$ increases from 0 to 1 , is similar to that described in section 4.1. However, the splitting of region CR4 (as in figure 2(b)) into two eddies (as in figure 2(a)) occurs at very small $H_{\mathrm{w}}$. In contrast, the second topological metamorphosis, (ii), is large-scale as described below.

\subsection{Development of clockwise circulation near the bottom center}

Figure 10(a) depicts the streamline pattern at $H_{\mathrm{w}}=0.52$. This pattern is topologically the same as that shown in figure 2(a), but eddies ME, CR3 and CR4 are too small to be visible in 
(a)

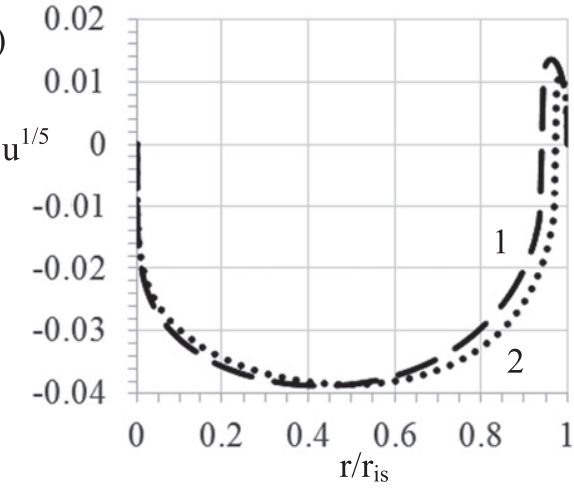

(b)

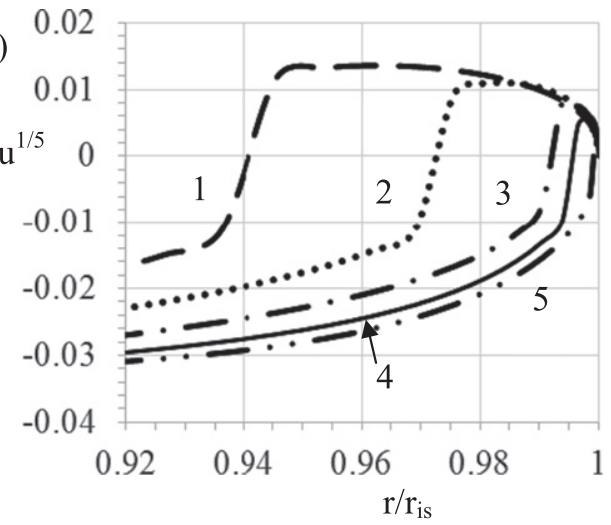

Figure 7. Distribution of radial velocity $u$ at the interface, $z=H_{\mathrm{w}}=0.05$, for $\alpha=90^{\circ}$ $(1), 100^{\circ}(2), 110^{\circ}(3), 120^{\circ}(4)$, and $130^{\circ}(5)$; (b) is a close-up of (a) near the sidewall, $r=r_{\text {is }}$.

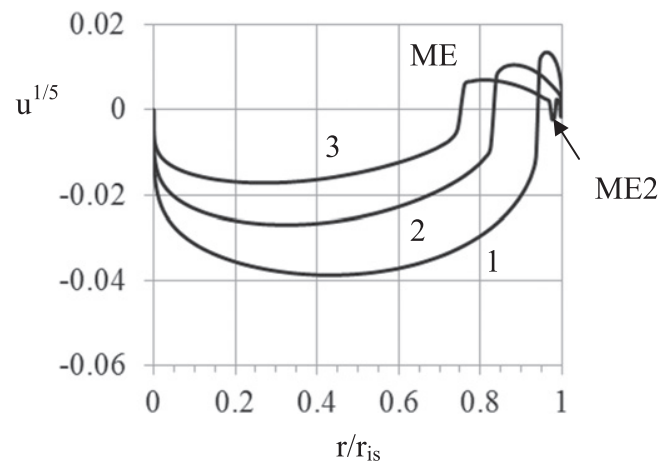

Figure 8. Distribution of radial velocity $u$ at the interface, $z=H_{\mathrm{w}}=0.05$, for $\alpha=90^{\circ}$ $(1), 70^{\circ}(2)$ and $60^{\circ}(3)$.

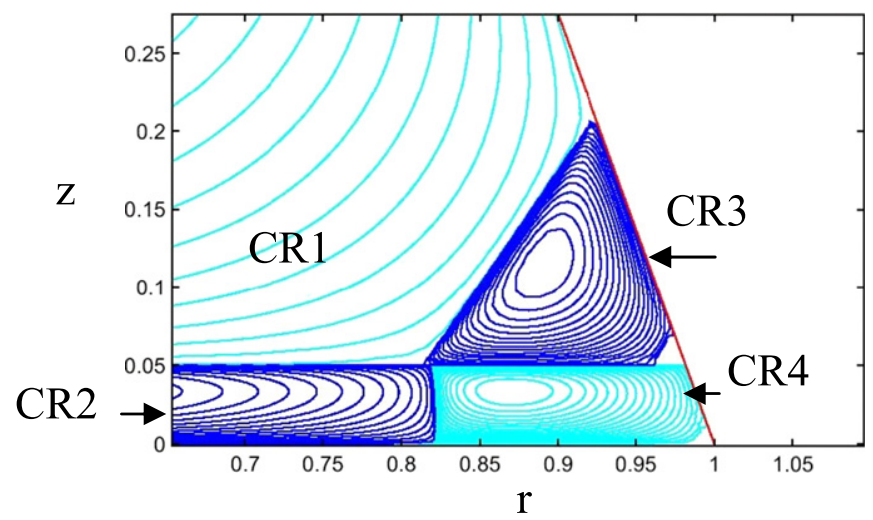

Figure 9. Pattern of meridional flow in bottom-sidewall corner at $H_{\mathrm{w}}=0.05$ and $\alpha=70^{\circ}$. 

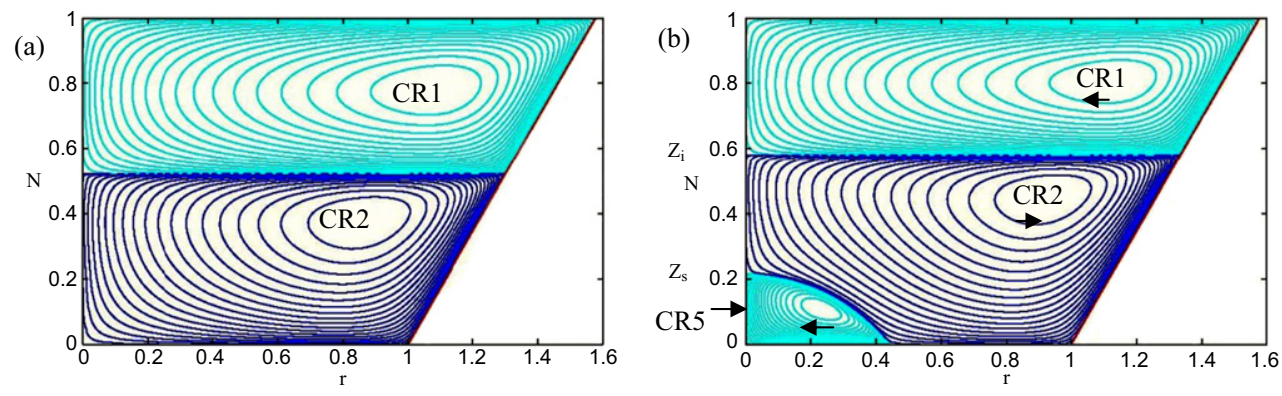

Figure 10. Vortex breakdown in water as $H_{\mathrm{w}}$ increases: $H_{\mathrm{w}}=0.52$ (a) and 0.58 (b); $\alpha=120^{\circ}$.

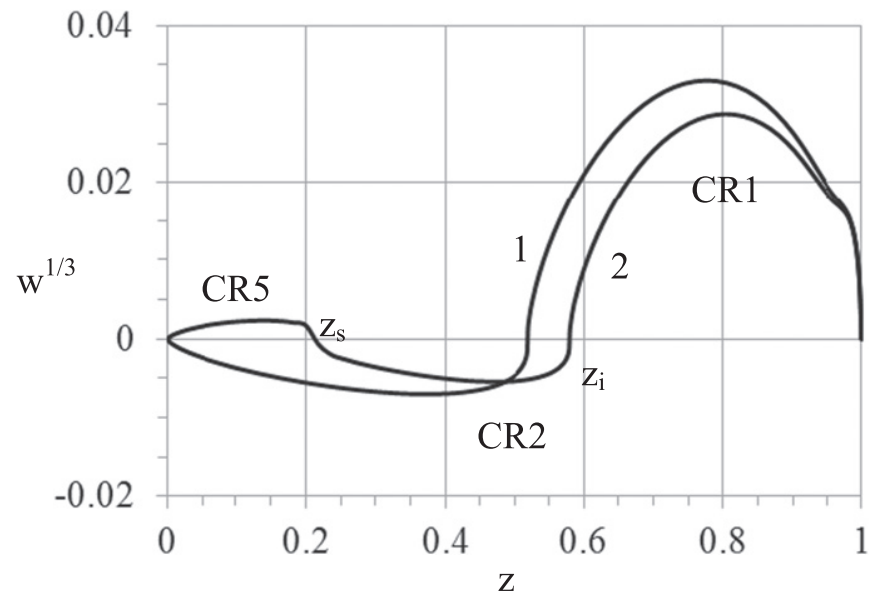

Figure 11. Distribution of velocity $w$ at the axis at $\alpha=120^{\circ} ; H_{\mathrm{w}}=0.52$ (1) and $0.58(2)$.
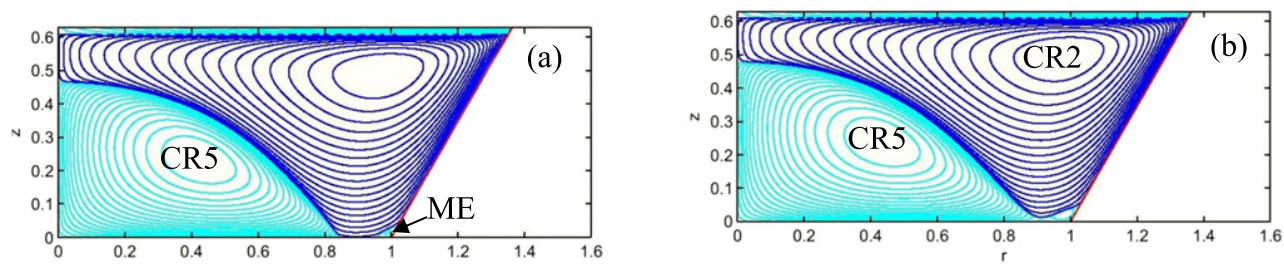

Figure 12. Merging of regions CR5 and ME, observed at $H_{\mathrm{w}}=0.608$ (a), into region CR5 at $H_{\mathrm{w}}=0.61$ (b) at $\alpha=120^{\circ}$.

figure 10. Figure 11 depicts the velocity, $w$, at the axis, $r=0$, at $H_{\mathrm{w}}=0.52$ (curve1) and 0.58 (curve 2). We plot $w^{1 / 3}$ in figure 11 to better observe small values of $w$ in the water region, $0<z<H_{\mathrm{w}}$. The $w>0$ range, $0<z<z_{\mathrm{s}}$, corresponds to region $\mathrm{CR} 5$ in figure 10(b); the $w<0$ range, $z_{\mathrm{s}}<z<z_{\mathrm{i}}$, corresponds to region CR2 in figure 10(b); and the $w>0$ range, $z_{\mathrm{i}}<z<1$, corresponds to region CR1 in air. 

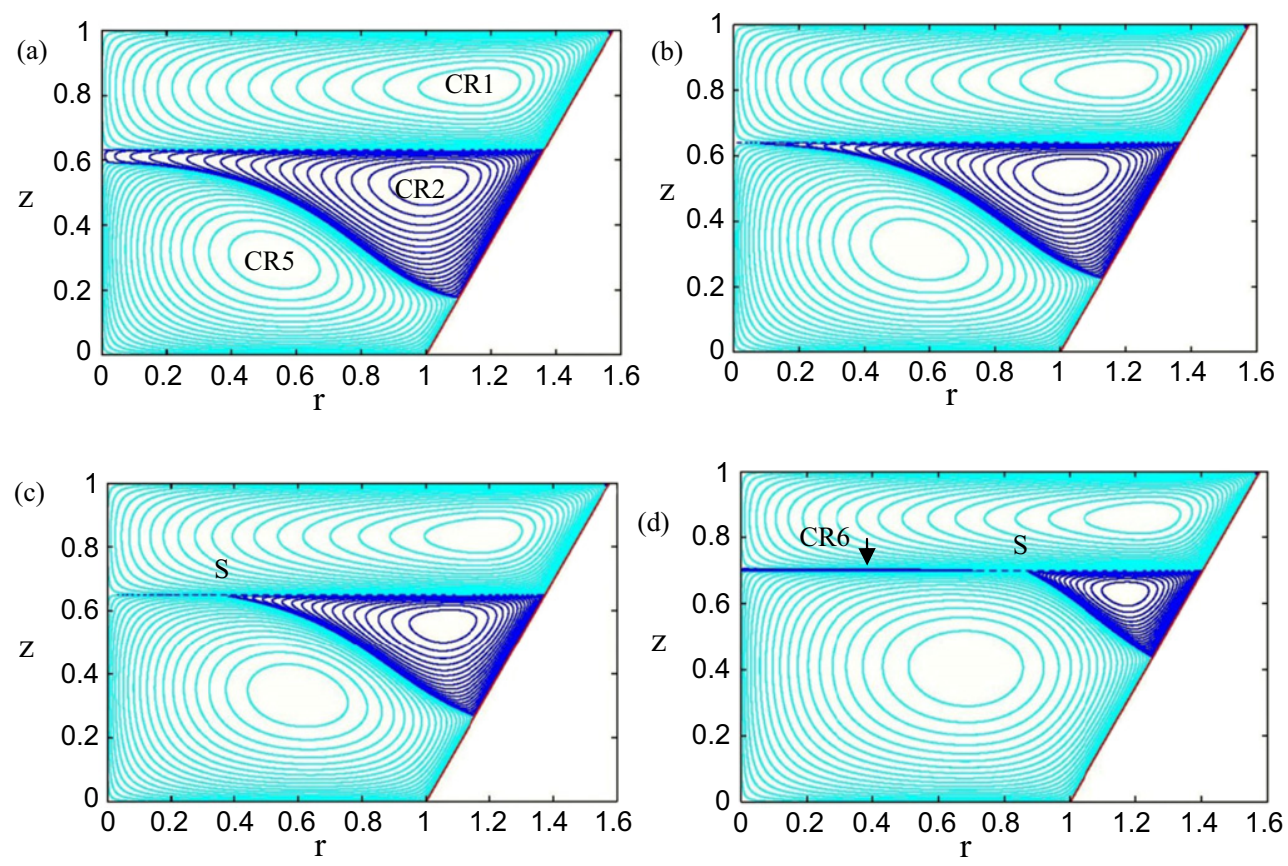

Figure 13. Emergence of vortex breakdown bubble (region CR6) in air at $\alpha=120^{\circ}$ as $H_{\mathrm{w}}$ increases: $H_{\mathrm{w}}=0.63(\mathrm{a}), H_{\mathrm{w}}=H_{\mathrm{w} 4}=0.648(\mathrm{~b}), H_{\mathrm{w}}=0.65(\mathrm{c})$ and 0.7 (d).

Interpolation yields that, as $H_{\mathrm{w}}$ decreases from $0.58, z_{\mathrm{s}}$ becomes zero at $H_{\mathrm{w}}=H_{\mathrm{ww}}=0.563$. Therefore at $H_{\mathrm{w}}=H_{\mathrm{ww}}$, VB occurs in the water flow at the axis-bottom intersection point, resulting in the emergence of region CR5. This second topology transformation, (ii), as $H_{\mathrm{w}}$ increases, is an example of a corner bifurcation, studied by Brøns (1994), being similar to that occurring in the cylindrical container (Herrada and Shtern 2014).

\subsection{Merging of near-bottom cells}

As $H_{\mathrm{w}}$ increases, region CR5 enlarges. Initially, region CR5 mostly extends in the radial direction and merges with the outmost ME; see ME in figure 12(a) (note that ME is visible here in contrast to figure 10). This is the third change in the flow topology, (iii), at $H_{\mathrm{w}}=0.609$. The bifurcation details are similar to those described by Brøns (2007) and Herrada et al (2013b, figure 5). They include bifurcation D8 (iv) in the merged region CR5.

\subsection{Development of thin circulation layer in air}

Next, region CR5 expands upward, reaches the interface at $H_{\mathrm{w}}=H_{\mathrm{wa}}=0.648$, and extends along the interface, reversing velocity $u$ at the interface near the axis. The water flow, corresponding to the $u>0$ range, drives the anticlockwise circulation of air in a thin circulation region, CR6. The CR6 emergence (VB in air) is the fifth change in the flow topology, (v). Figure 13 depicts the corresponding transformation of streamline pattern.

Region CR2 topologically is a bubble at $H_{\mathrm{w}}=0.63$ (figure 13(a)), becomes a bubblering at $H_{\mathrm{w}}=H_{\mathrm{w} 4}=0.648$ (figure $13(\mathrm{~b})$ ), and is a ring in figure $13(\mathrm{c})\left(H_{\mathrm{w}}=0.65\right.$ ) and figure $13(\mathrm{~d})\left(H_{\mathrm{w}}=0.7\right)$. Region CR6 is very thin at $H_{\mathrm{w}}=0.65$ and clearly visible at $H_{\mathrm{w}}=0.7$ (figure 13(d)). Regions CR2 and CR6 touch each other at the saddle point, $S$, where 


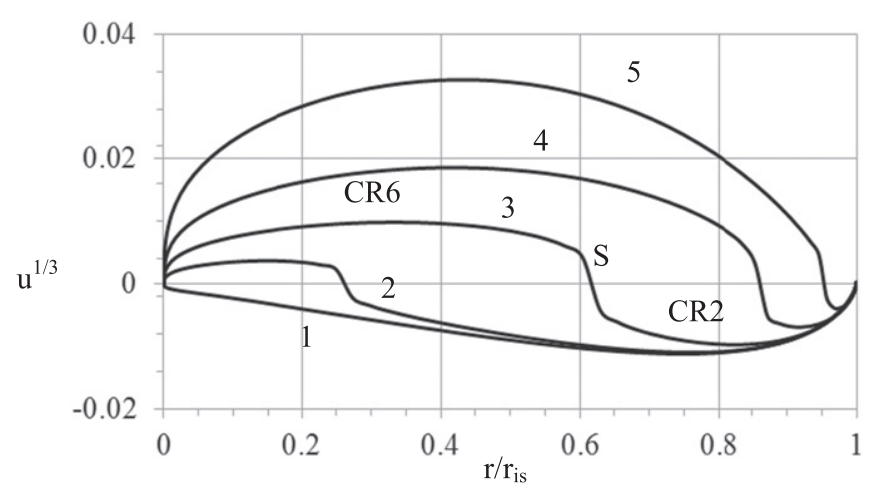

Figure 14. Distribution of radial velocity $u$ at the interface at $\alpha=120^{\circ}$ and $H_{\mathrm{w}}=0.64$ (1), 0.65 (2), 0.7 (3), 0.8 (4) and 0.9 (5). Saddle $S$ separates regions CR6 and CR2.

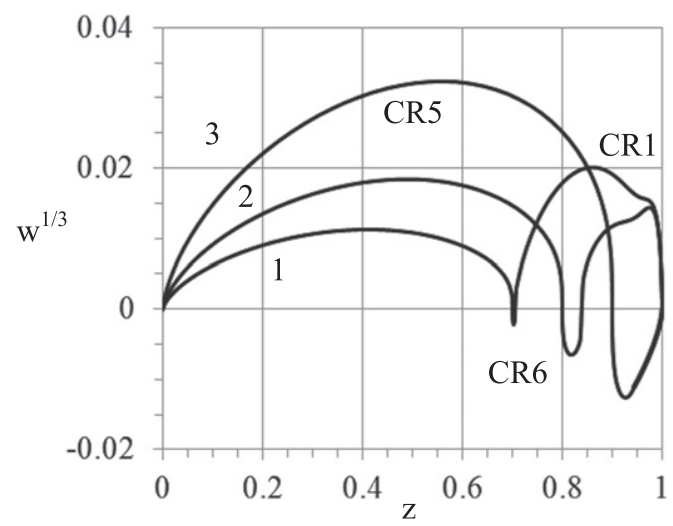

Figure 15. Distribution of velocity $w$ on the axis at $\alpha=120^{\circ}$ and $H_{\mathrm{w}}=0.7$ (1), 0.8 (2), and $H_{\mathrm{w}}=H_{\mathrm{w} 5}=0.867$ (3). The $w<0$ range corresponds to region CR6.

regions CR1 and CR5 also touch each other; $S$ is a stagnation point of the meridional motion where $u=w=0$, but $v \neq 0$. As $H_{\mathrm{w}}$ increases, region CR6 rapidly expands in the radial direction as figure 14 illustrates where the $u>0(u<0)$ ranges correspond to region CR6 (CR2).

The emergence of region CR6 is a topological metamorphosis similar to that which occurs in the cylindrical container (Herrada and Shtern 2014), but here the development of CR6, as $H_{\mathrm{w}}$ further increases, is very different: the $z$-extent of CR6, $z_{\mathrm{s}}-z_{\mathrm{i}}$, monotonically grows with $H_{\mathrm{w}}$, as figure 15 depicts. In figure 15 , velocity $w$ at the axis is presented at $H_{\mathrm{w}}=0.7$ (1), $0.8(2)$, and $H_{\mathrm{w}}=H_{\mathrm{w} 5}=0.867$ (3). To better observe small $w<0$ intervals, $z_{\mathrm{i}}<z<z_{\mathrm{s}}$, corresponding to region CR6, we plot $w^{1 / 3}$ in figure 15 . The $w>0$ interval, $0<z<z_{\mathrm{i}}\left(z_{\mathrm{s}}<z<1\right)$ corresponds to region CR5 (CR1).

\subsection{Separation of region $C R 1$ from the axis}

As the expanding region CR6 reaches the rotating top disk at $H_{\mathrm{w}}=H_{\mathrm{w} 5}=0.867$, the sixth change of the flow topology, (vi), occurs. Region CR1 separates from the axis and topologically becomes a ring as figure 16 illustrates depicting streamline patterns at $H_{\mathrm{w}}=0.86$ (a), 

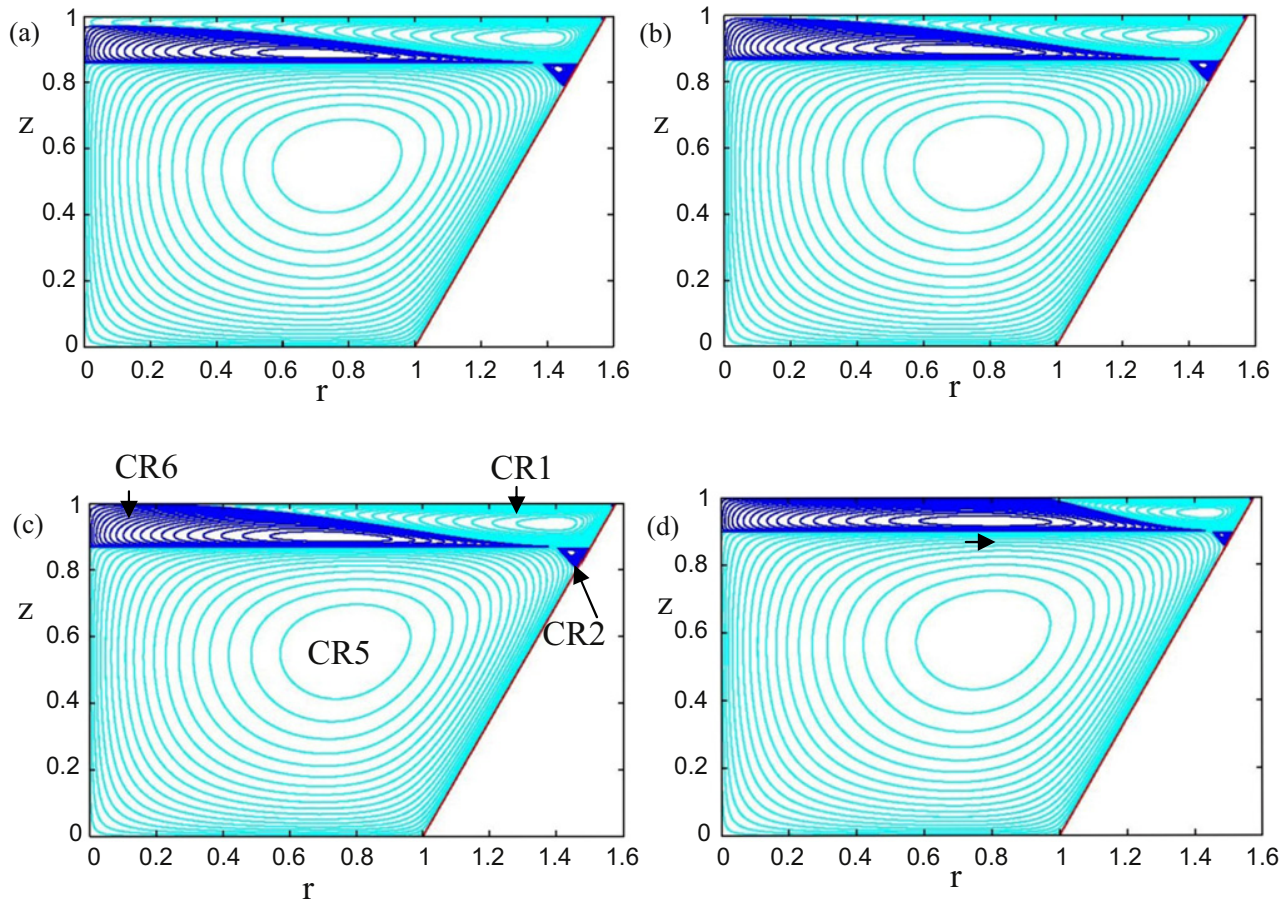

Figure 16. Separation of region 1 from the axis at $\alpha=120^{\circ}$ as $H_{\mathrm{w}}$ increases: $H_{\mathrm{w}}=0.86(\mathrm{a}), H_{\mathrm{w}}=H_{\mathrm{w} 5}=0.867(\mathrm{~b}), H_{\mathrm{w}}=0.87(\mathrm{c})$ and $0.9(\mathrm{~d})$.

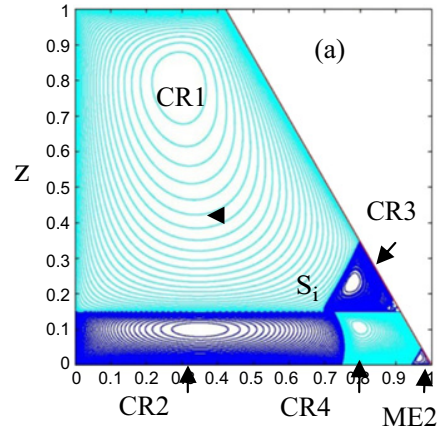

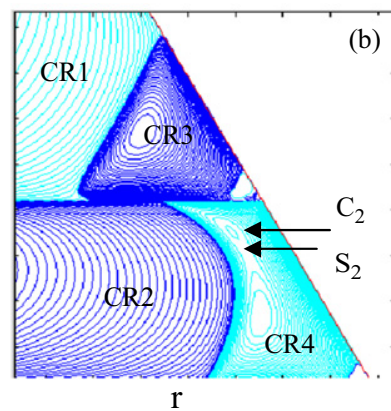

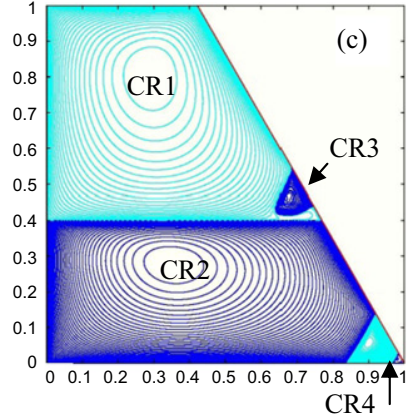

Figure 17. Expansion of region CR2 up to sidewall and separation of region CR3 from the interface as $H_{\mathrm{w}}$ increases: $H_{\mathrm{w}}=0.15$ (a), 0.21 (b) and 0.4 (c); $\alpha=60^{\circ}$.

$H_{\mathrm{w}}=H_{\mathrm{w} 5}=0.867(\mathrm{~b}), H_{\mathrm{w}}=0.87$ (c) and 0.9 (d). This is again an example of a corner bifurcation (Brøns 2007). After the separation from the axis, region CR1 shrinks toward the sidewall-top-disk corner as $H_{\mathrm{w}}$ further increases (figure 16(d)). No further changes in the flow topology occur as $H_{\mathrm{w}}$ approaches 1 .

It is an interesting physical feature that the air mostly circulates in the anticlockwise direction (figure 16(d)) despite the fact that the centrifugal force, induced by the rotating disk, tends to move air in the clockwise direction. The feedback of the rotating water flow 
(a)

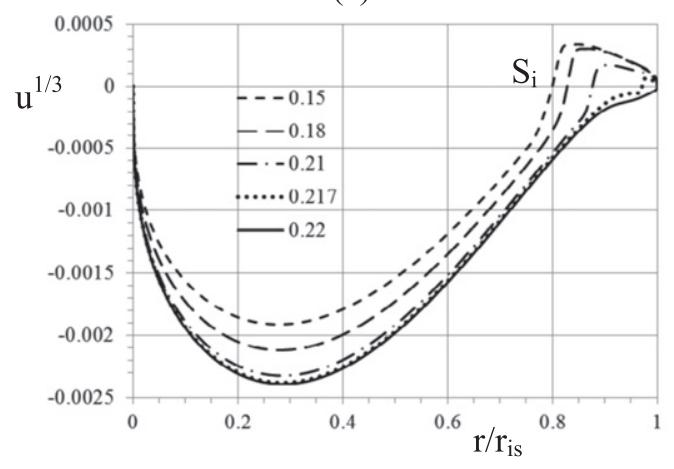

(b)

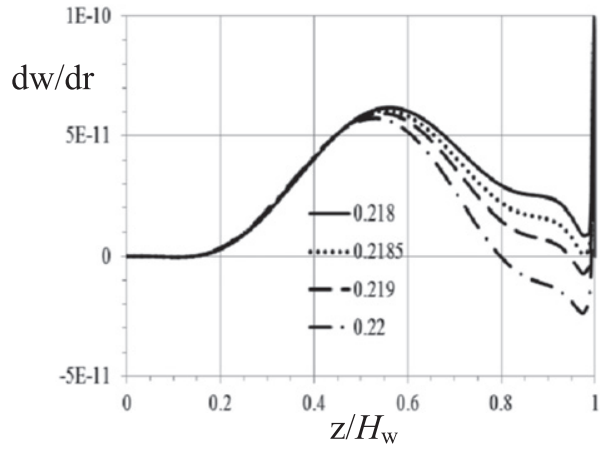

Figure 18. (a) Profile of radial velocity $u$ at the interface and (b) dependence of $\mathrm{d} w / D r$ on $z$ at the sidewall in water at $H_{\mathrm{w}}$ values shown in the picture. Saddle $S_{\mathrm{i}}$ separates the $u<0$ and $u>0$ ranges.
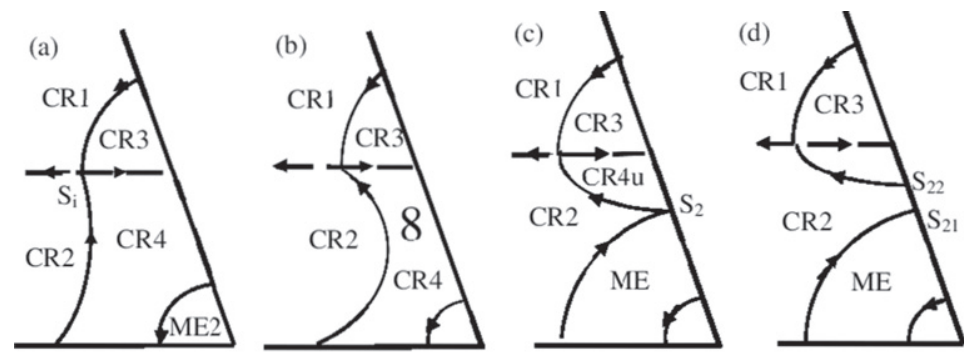

(e)
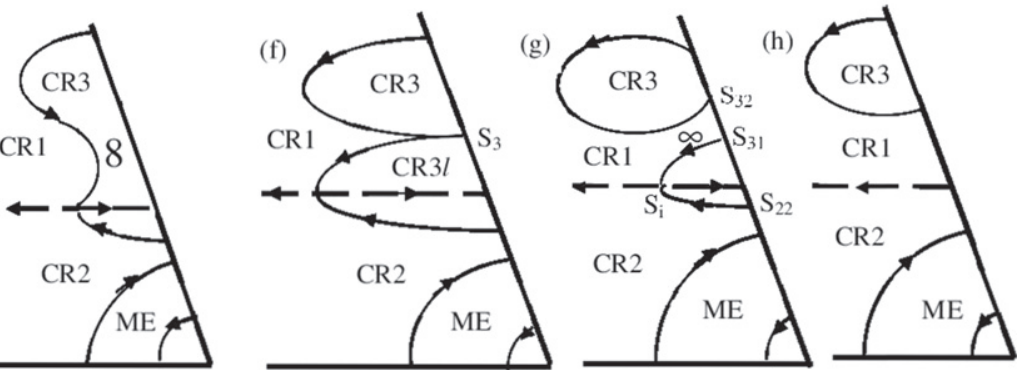

Figure 19. Schematics showing transformation of figure 17(a) into figure 17(c). For clarity, figure-eight structures are not shown.

overcomes the direct effect of the centrifugal force which pushes the air to the periphery near the disk.

The topological transformations, reported in this section, significantly differ from those in the cylindrical device (Herrada and Shtern 2014). As $H_{\mathrm{w}}$ increases from 0 to 1 , six (ten) changes of flow topology occur in the conical (cylindrical) container. VBs in the water (at $H_{\mathrm{w}}=0.563$ ) and in the air (at $H_{\mathrm{w}}=0.648$ ) occur at smaller values of $H_{\mathrm{w}}$ than those in the cylindrical case. These features are due to the sidewall here converges from the rotating disk to the stationary disk and thus strengthens the effect of swirl on the water and air motions. Now we explore the effect of a diverging sidewall. 


\section{Topological flow metamorphoses in the $\alpha=60^{\circ}$ cone as $H_{w}$ increases}

\subsection{Expansion of region CR2 up to the sidewall}

Figure $17(\mathrm{a})$ is a streamline pattern at $H_{\mathrm{w}}=0.15$. This pattern is topologically the same as shown in figure 9. No topological change occurs as $H_{\mathrm{w}}$ decreases from 0.15 down to zero. In contrast as $H_{\mathrm{w}}$ increases, numerous changes occur in the flow topology. To clarify the transformations shown in figure 17, we explore the dependence on $H_{\mathrm{w}}$ of the distribution of radial velocity $u$ on the interface. To better observe the $u>0$ region where $u$ is small, figure 18(a) depicts $u^{1 / 3}$.

The $u<0(u>0)$ range in figure 18(a) corresponds to the boundary between regions CR1 and CR2 (CR3 and CR4), see figure 17(a). It follows from figure 18(a) that regions CR1, CR2, CR3, and CR4 meet at one point $S_{\mathrm{i}}$ (figures 17(a) and 18(a)) which is a saddle stagnation point of meridional motion as figure 19(a) schematically shows. (The accuracy of drawing in figure 17(a) is not sufficient to clearly show that regions CR2 and CR3 touch each other only at a single point $-S_{\mathrm{i}}$.) Figure $18(\mathrm{a})$ also shows that $S_{\mathrm{i}}$ shifts toward the sidewall as $H_{\mathrm{w}}$ increases, i.e., the radial coordinate $r_{\mathrm{s}}$ of $S_{\mathrm{i}}$ approaches $r_{\text {is }}$.

Figure 17(b) depicts streamlines at $H_{\mathrm{w}}=0.21$ near the bottom-sidewall intersection and reveals that the water flow has a figure-eight pattern in region CR4, as figure 19(b) schematically shows. Therefore as $H_{\mathrm{w}}$ increases and CR4 shrinks to the sidewall, (i) center $\mathrm{C}_{2}$ and saddle $S_{2}$ (figure 17(b)) both emerge via a cusp catastrophe (A8). Then saddle $S_{2}$ moves to the sidewall, reaches it at $H_{\mathrm{w}}=H_{\mathrm{w} 2}$ (figure 19(c)), and (ii) splits into two saddles $S_{21}$ and $S_{22}$ (figure 19(d)). As a result, region CR2 extends up to the sidewall and region CR4 becomes divided into CR4u and ME (figure 19(c)). This occurs at $H_{\mathrm{w}}=H_{\mathrm{w} 1}=0.219$ at $\alpha=60^{\circ}$ (compare with $H_{\mathrm{w} 1}=0.078$ at $\alpha=90^{\circ}$ ).

Figure 18(b) confirms this bifurcation scenario by depicting the dependence of $\mathrm{d} w / \mathrm{d} r$ on $z$ at the sidewall in water at a few $H_{\mathrm{w}}$ values shown in the picture. At $H_{\mathrm{w}}=0.218$ and 0.2185 , $\mathrm{d} w / \mathrm{d} r$ is positive in the $z$ range corresponding to region CR4 in figure 19(a). The local minimum of $\mathrm{d} w / \mathrm{d} r$ near the sidewall decreases and becomes negative as $H_{\mathrm{w}}$ increases. At $H_{\mathrm{w}}=0.219$ and 0.22 , the $z$ range near the interface, $z / H_{\mathrm{w}}=1$, where $\mathrm{d} w / \mathrm{d} r<0$, corresponds to region CR2 in figure 19(d). The physical reason for the splitting of region CR4 into two parts (figures 19 (a)-(d)) is that the water flow in region CR2 intensifies, as $H_{\mathrm{w}}$ increases, and pushes region CR4 to the sidewall. In region CR1 near the interface, the air flow also intensifies, pushes region CR3 to the sidewall, and splits it in two parts as described below.

\subsection{Separation of region CR3 from the interface}

Though saddle $S_{\mathrm{i}}$ is pressed to the sidewall by the strengthening flow (figure 18(a)), the presence of the interface-sidewall eddies (Shtern 2014) decelerates the $S_{\mathrm{i}}$ shift. Since the boundary between regions CR1 and CR3 is less resistant, the air flow makes it concave, resulting in (iii) the development of the figure-eight streamline pattern in CR3 (figure 19(e)) via the cusp catastrophe (A8). Next, the figure-eight saddle $S_{3}$ approaches the sidewall, touches it (figure 19(f)) and (iv) splits into saddles $S_{31}$ and $S_{32}$ (figure 19(g)). As a result, region CR3 becomes divided into regions CR3 and CR3l (figures 19(f) and (g)).

The vortices CR3l and CR4u (figure 19) shrink to the interface-sidewall intersection point and become unresolved (figure 19(h)) with our grid as $H_{\mathrm{w}}$ further increases (figures 17(c) and 19(h)). However they should exist according to the theory of interfacesidewall eddies (Shtern 2014). 

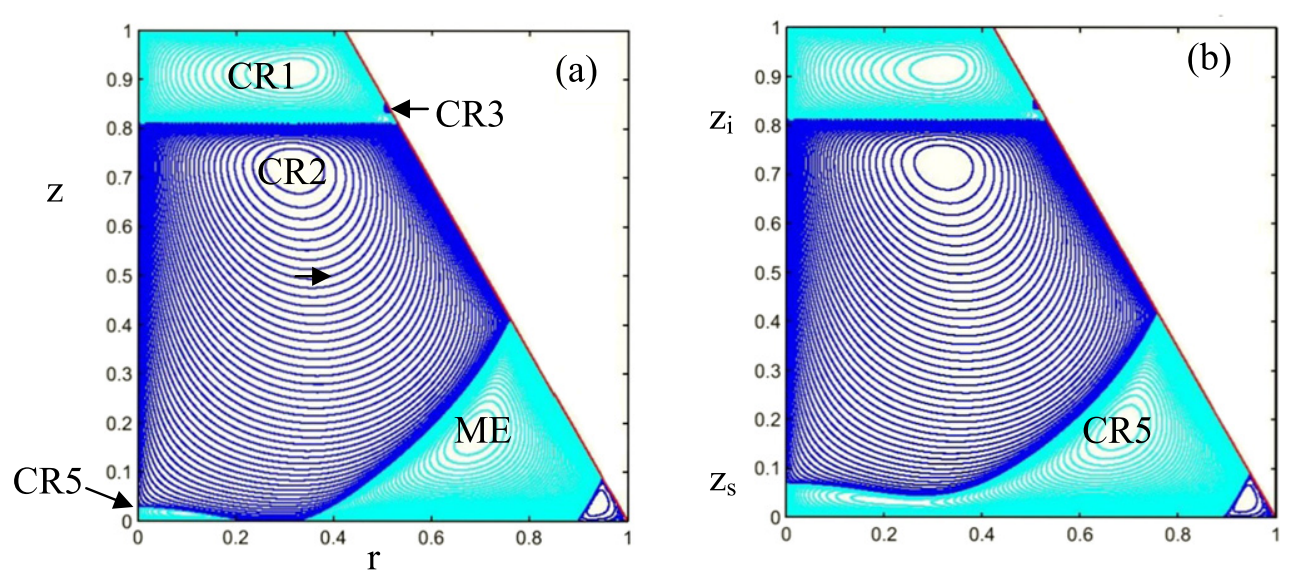

Figure 20. Appearance of region CR5 and its mergence with region $\mathrm{ME}$ as $H_{\mathrm{w}}$ increases: $H_{\mathrm{w}}=0.81115$ (a) and 0.81119 (b); $\alpha=60^{\circ}$.

Just after region CR3 separates from the interface, a part of region CR1 has a thin 'neck' located between the interface and region CR3 (see figures 17(c) and 19(g)). Therefore the figure-eight pattern develops in region $\mathrm{CR} 1$ in the vicinity of the sidewall between the interface and region CR3 (figure 19(g)). This occurs via (v) the cusp catastrophe (A8). With increasing $H_{\mathrm{w}}$, the gap between the interface and region CR3 becomes larger and a pattern reversal occurs at $H_{\mathrm{w}} \approx 0.37$ : the figure-eight pattern disappear (figure 19(h)) via (vi) a cusp catastrophe (D8). This event finalizes the streamline transformation from that shown in figure 17 (a) to that shown in figure $17(\mathrm{c})$.

\subsection{VB in the water flow}

The streamline pattern, depicted in figure $17(\mathrm{c})$, remains topologically invariant as $H_{\mathrm{w}}$ increases until it reaches a value around 0.81 . At $H_{\mathrm{w}}$ slightly smaller than 0.81 , a cell with clockwise circulation emerges near the axis-bottom intersection (region CR5 in figure 20(a)), i.e., VB occurs in the water flow.

Since region ME significantly expands as $H_{\mathrm{w}}$ increases from 0.4 to 0.81 (compare figures 17(c) and 20(a)), regions CR5 and ME merge just after CR5 appearance, as figure 20(b) illustrates. The merged region is again named CR5. Note that region CR3 shrinks but remains observable in figure 20 .

The transformations from figure 17(c) through the patterns shown in figure 20 consists of the following topological events: (vii) VB emergence near the axis-bottom intersection, (viii) merging of the near-axis saddles at the bottom, followed by separation of the merged saddle from the bottom, and (ix) D8 in region CR5.

\section{4. $V B$ in the air flow}

Interpolation yields that region CR5 emerges at $H_{\mathrm{w}}=0.8098$, where $z_{\mathrm{s}}=0$. Region CR5 rapidly expands upward as $H_{\mathrm{w}}$ increases and touches the interface, $z=z_{\mathrm{i}}=H_{\mathrm{w}}$, at $H_{\mathrm{w}}$ being very close to 0.85 where $z_{\mathrm{s}}=z_{\mathrm{i}}$. The function $z_{\mathrm{s}}\left(H_{\mathrm{w}}\right)$ is nearly linear in the vicinity of $H_{\mathrm{w}}=0.81$ and therefore the interpolation works well.

Figure 21 depicts the streamline patterns for (a) $z_{\mathrm{s}}<z_{\mathrm{i}}\left(H_{\mathrm{w}}=0.84\right)$ and (b) $z_{\mathrm{s}}>z_{\mathrm{i}}$ $\left(H_{\mathrm{w}}=0.85\right)$. As the clockwise circulation in region CR5 reaches the air, it reverses the radial 

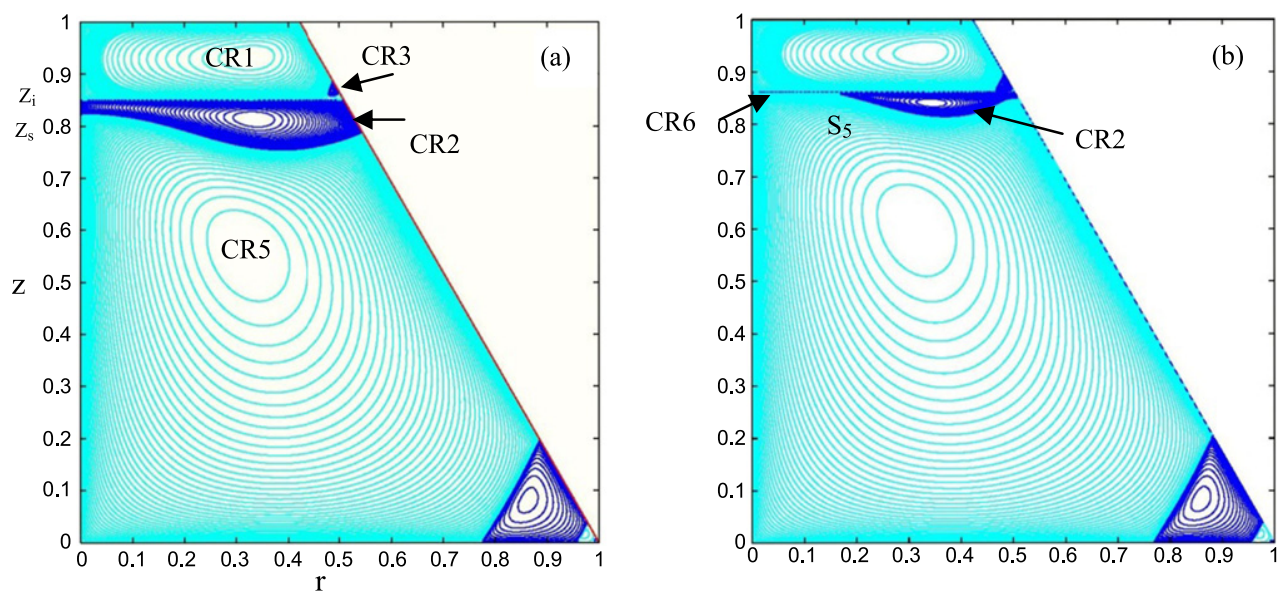

Figure 21. Streamline patterns for $z_{\mathrm{s}}$ close to $z_{\mathrm{i}}:$ (a) $z_{\mathrm{s}}<z_{\mathrm{i}}\left(H_{\mathrm{w}}=0.84\right)$ and (b) $z_{\mathrm{s}}>z_{\mathrm{i}}$ $\left(H_{\mathrm{w}}=0.85\right) ; \alpha=60^{\circ}$.

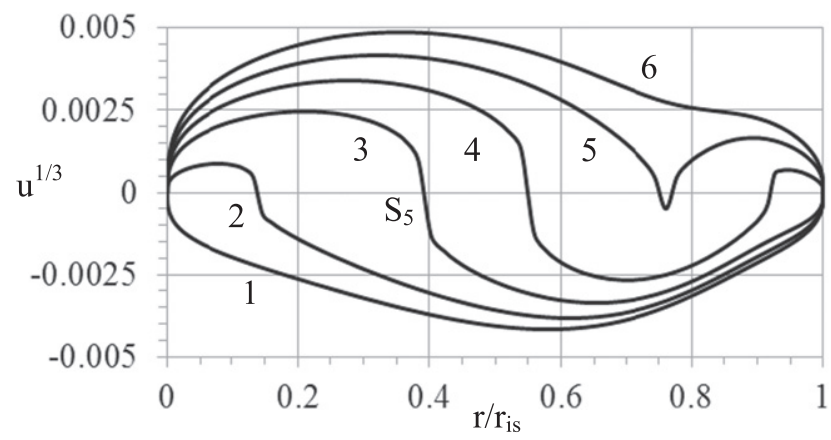

Figure 22. Distribution of radial velocity $u$ at the interface at $\alpha=60^{\circ}$ and $H_{\mathrm{w}}=0.84$ (1), 0.85 (2), 0.86 (3), 0.87 (4), 0.88 (5), and 0.89 (6). Saddle $S_{5}$ separates regions CR6 and CR2.

velocity at the interface, which becomes positive, and drives an anticlockwise circulation in the thin air region CR6 (figure 21(b)). Figure 22 presents the distribution of radial velocity $u$ at the interface for $H_{\mathrm{w}}=0.84$ (curve 1), 0.85 (curve 2), 0.86 (curve 3), 0.87 (curve 4), 0.88 (curve 5) and 0.89 (curve 6).

Figure 22 depicts $u^{1 / 3}$ in order to better observe regions, where $|u|$ is small. For the abscissa, the ratio, $r / r_{\text {is }}$, is used. This allows for convenient comparison of curves in figure 21 .

At $H_{\mathrm{w}}=0.84, u<0$ for $0<r<r_{\text {is }}$, which means that region CR2 is attached to the entire interface as it appears from curve 1 in figure 22(a). For all $H_{\mathrm{w}}>0.84$, there is a region $0<r<r_{\mathrm{s}}$, where $u>0$. Therefore there must be a new region CR6 in the air. This region is attached to the interface and the air circulation in CR6 is anticlockwise. Here $r_{\mathrm{s}}$ is the radial coordinate of saddle point $S_{5}$ (see figures 21(b), 22 and 24(a)) where regions CR2 and CR6 


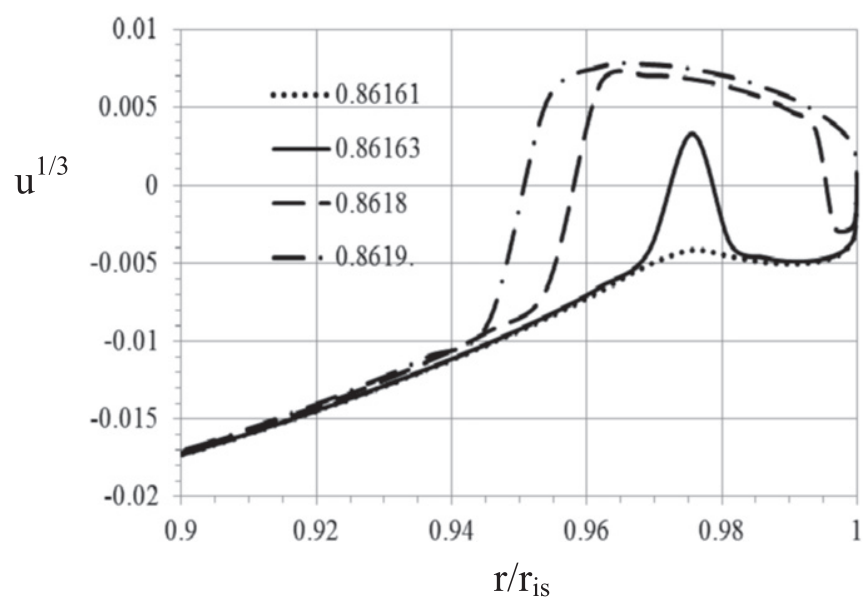

Figure 23. Distribution of radial velocity $u$ on the interface near the sidewall for $H_{\mathrm{w}}$ values shown in the picture.
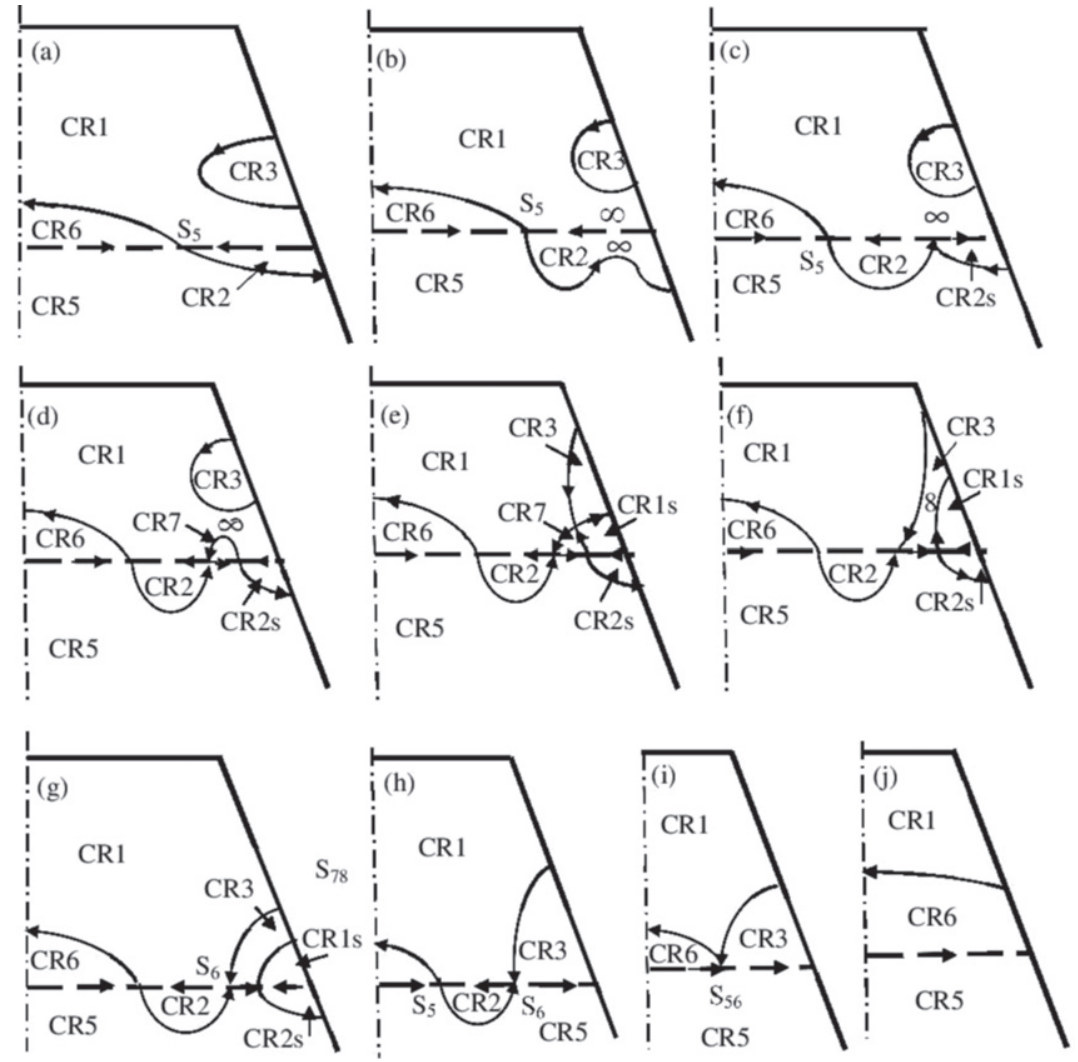

Figure 24. Schematics showing reattachment of region CR3 to the interface and collapse of region CR2. 
meet. A striking feature is that region CR6 is a very thin layer. For example at $H_{\mathrm{w}}=0.87$, its radial extent is $r_{\mathrm{s}} / r_{\mathrm{is}}=0.56$ while its axial thickness is $z_{\mathrm{s}}-z_{\mathrm{i}}=0.0017$.

The physical reason for the VB development in the air and the shrinking of region CR2 in the water is the strengthening of water swirl as the interface approaches the rotating top disk. The growing centrifugal force intensifies the clockwise circulation in region CR5 and suppresses the anticlockwise circulation in region CR2. The air flow is affected by the water flow feedback. This causes further topological metamorphoses in both water and air flows.

The CR6 emergence is the tenth (x) topological event. Curve 4 in figure 22 also has the $u>0$ range near the sidewall, $r=r_{\text {is }}$. This feature indicates more topological transformations discussed below.

\subsection{Reattachment of region $\mathrm{CR} 3$ to the interface}

Figure 23, being a close up of figure 22 near the sidewall, reveals a local reversal of $u$ at the interface as $H_{\mathrm{w}}$ increases. The dotted curve $\left(H_{\mathrm{w}}=0.86161\right)$ in figure 23 has a local maximum near $r / r_{\text {is }}=0.975$. As this maximum approaches the line $u=0$ in figure 23, (a) the adjacent water flow decelerates causing (xi) A8, i.e. the appearance of a figure-eight pattern in region CR2, and (b) the adjacent air flow decelerates causing (xii) A8, i.e. the appearance of a figure-eight pattern in region CR1 between the interface and region CR3. Figures 24(a) and (b) schematically show the corresponding transformations of the flow pattern.

Figure 23, being a close up of figure 22 near the sidewall, reveals a local reversal of $u$ at the interface as $H_{\mathrm{w}}$ increases. The dotted curve $\left(H_{\mathrm{w}}=0.86161\right)$ in figure 23 has a local maximum near $r / r_{\text {is }}=0.975$. As this maximum approaches the line $u=0$ in figure 23 , (a) the adjacent water flow decelerates causing (xi) A8, i.e. the appearance of a figure-eight pattern in region CR2, and (b) the adjacent air flow decelerates causing (xii) A8, i.e. the appearance of a figure-eight pattern in region CR1 between the interface and region CR3. Figures 24(a) and (b) schematically show the corresponding transformations of the flow pattern.

A small increment of $H_{\mathrm{w}}$ to 0.86163 (solid curve in figure 23) yields an internal part of the interface with $u>0$ and a streamline pattern as shown schematically in figure $24(\mathrm{~g})$. The narrow range, $0.86161<H_{\mathrm{w}}<0.86163$, has not been numerically resolved. It is not possible to transform pattern (b) into (g) through a single bifurcation. The scenario, we propose in figure 24 is the simplest one possible and is based on the theoretical analysis by Brons (1995, figure 5) and the physical reason that the water (air) can (cannot) easily reverse the air (water) flow. This suggests the following steps: the figure-eight saddle in the water touches the interface at $H_{\mathrm{w}}=0.86162$ and region CR2s emerges from a part of CR2 (figure 24(c)). As $H_{\mathrm{w}}$ further increases, the saddle splits into two saddles both located at the interface (xiii) and region CR7 emerges in the air (figure 24(d)). Next, (xiv) regions CR3 and CR7 touch each other at the figure-eight saddle (figure 24(e)) and merge (figure 24(f)). We denote the merged region again as CR3.

After the merging, CR3 has the eight-figure pattern in its neck part (figure 24(f)). Next, the figure-eight saddle and the lower center point merge and disappear via (xv) cusp catastrophe D8 (figure 24(g)). As $H_{\mathrm{w}}$ further increases, regions CR1s and CR2s together shrink toward the interface-sidewall intersection point and becomes unresolved by our grid (as figure 24(h) schematically illustrates) though they should exist (Shtern 2014). 

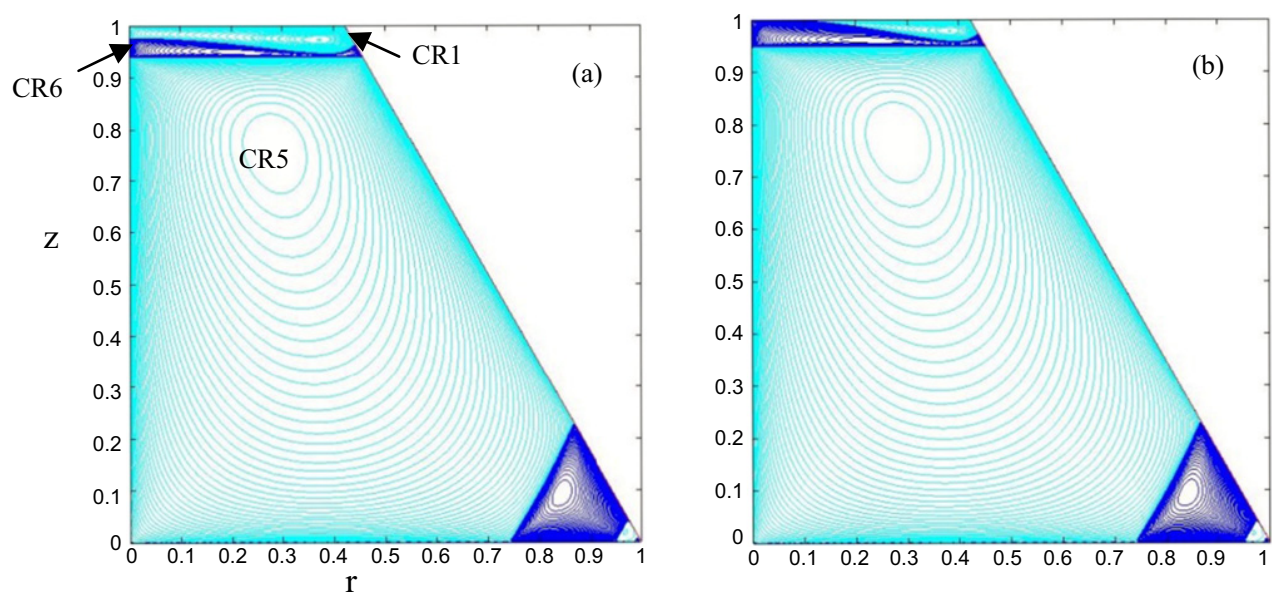

Figure 25. Streamline patterns at (a) $H_{\mathrm{w}}=0.94$ and (b) $H_{\mathrm{w}}=0.951 ; \alpha=60^{\circ}$.

\subsection{Collapse of region $C R 2$}

Next, (xvi) saddles $S_{5}$ and $S_{6}$ (figure 24(h)) merge into saddle $S_{56}$ (figure 24(i)) which separates from the sidewall and (xvii) disappears via D8 resulting in mergence of regions CR3 and CR6 into one region again named as CR6 (figure 24(j)). These tranformations make region $\mathrm{CR} 6$ adjacent to the entire interface and region $\mathrm{CR} 1$ separated from the interface (figure 24(j)).

\subsection{Separation of region CR1 from the axis}

After region CR6 extends up to the sidewall (figure 24(j)), CR6's thickness at the axis, $z_{\mathrm{s}}-z_{\mathrm{i}}$, remarkably increases with $H_{\mathrm{w}}$, and $z_{\mathrm{s}}$ reaches 1 (i.e., the rotating disk) at $H_{\mathrm{w}}=0.95$. This (xviii) corner bifurcation results in the separation of region CR1 from the axis as figure 25 illustrates depicting the streamline pattern at (a) $H_{\mathrm{w}}=0.94$ (before the bifurcation) and at $H_{\mathrm{w}}=0.951$ (after the bifurcation). Finally, region CR6 shrinks to the rotating-disk-sidewall intersection point as $H_{\mathrm{w}}$ approaches 1 .

The topological transformations, reported in section 6, also significantly differ from those in the cylindrical device (Herrada and Shtern 2014). As $H_{\mathrm{w}}$ increases from 0 to 1 , eighteen (ten) changes of flow topology occur in the conical (cylindrical) container. VBs in the water (at $H_{\mathrm{w}}=0.81$ ) and in the air (at $H_{\mathrm{w}}=0.85$ ) occur at larger values of $H_{\mathrm{w}}$ than those in the cylindrical case. These features are due to that the sidewall divergence from the rotating disk to the still disk weakens the effect of swirl on the water and air motions.

\section{The effect of increasing the value of the Reynolds number}

To explore in what range of the Reynolds number, $R e$, the flow topology remains the same as that for the creeping flows, we come back from the reduced equations (section 2.4) to the full equations (sections 2.2 and 2.3) and use numerical techniques for the nonlinear problem (section 3).

To characterize the strength of air flow, we introduce the Reynolds number based on the air viscosity, $R e_{\mathrm{a}}=\omega R^{2} / \nu_{\mathrm{a}}=R e / \nu_{n}$, because the rotating top disk directly (indirectly) drives 

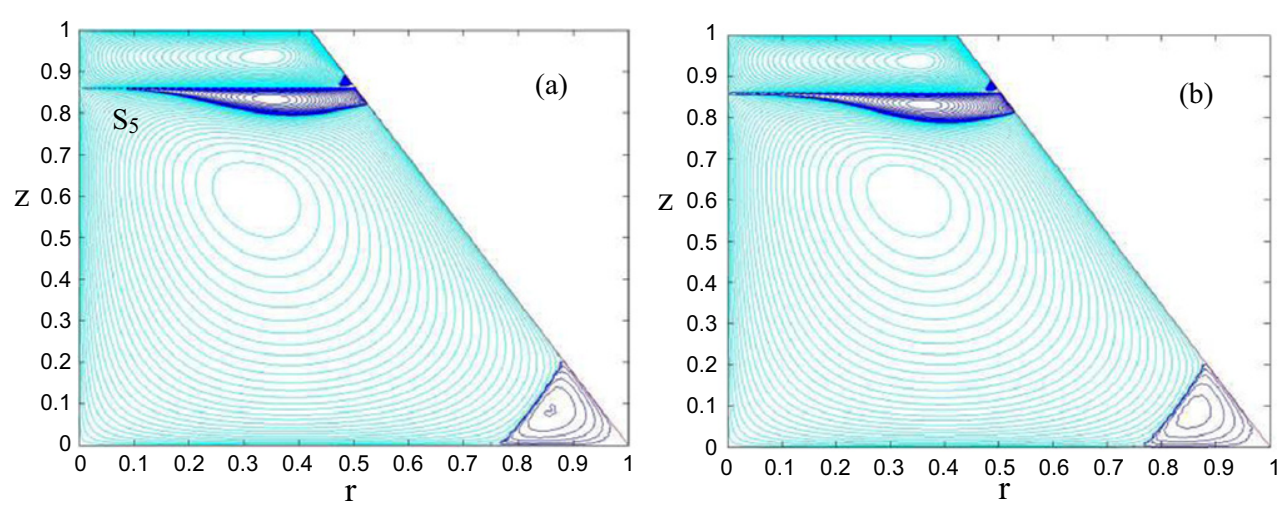

Figure 26. Streamline $H_{\mathrm{w}}=0.86 ; \alpha=60^{\circ}$.
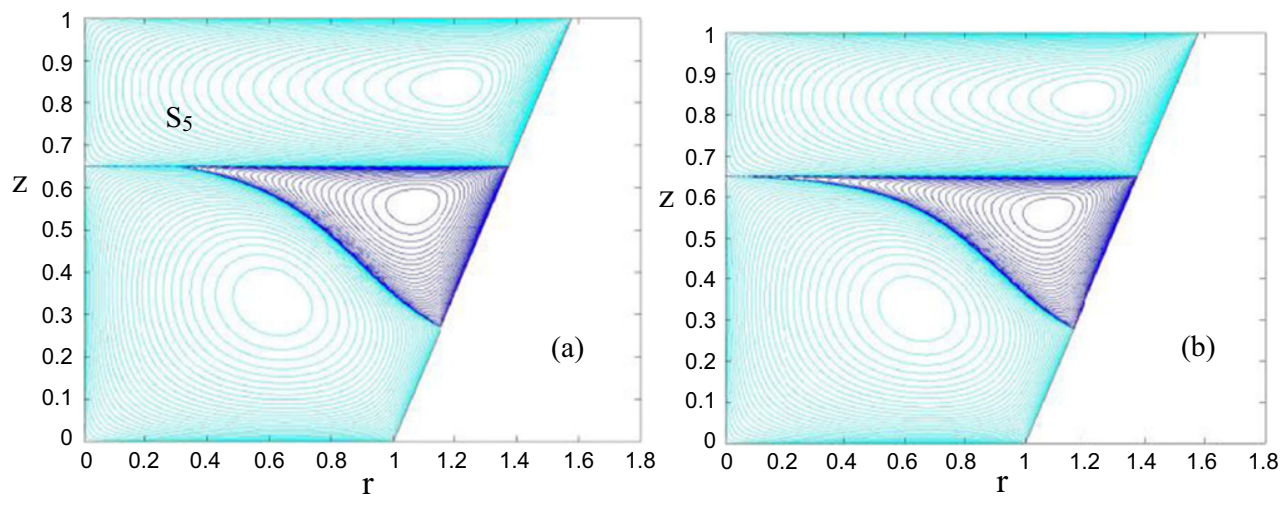

Figure 27. Streamline patterns at $R e=1000$ (a) and 2000 (b), $H_{\mathrm{w}}=0.65 ; \alpha=120^{\circ}$.

the air (water) flow; here we take $\nu_{n}=15$. From the top disk down to the interface, the swirl velocity significantly drops. Therefore, to characterize the strength of water flow, we introduce the Reynolds number, $R e_{\mathrm{w}}$, based on the maximal swirl velocity at the interface, $v_{\mathrm{im}}$; $R e_{\mathrm{w}}=v_{\mathrm{im}} R e$.

\subsection{Topological flow metamorphoses in the $\alpha=60^{\circ}$ cone as Re increases}

Figure 26 depicts the streamline patterns at $R e=10000$ (a) and 20000 (b) for $H_{\mathrm{w}}=0.86$ and $\alpha=60^{\circ}$. At $R e=10000$ (figure 26(a)), the flow topology is the same as in the creeping flow (figure 21(b)). The quantitative difference is that the radial extent, $r_{\mathrm{S}}$, of layer CR6 becomes smaller at $R e_{\mathrm{a}}=666$ than those for $R e_{\mathrm{a}} \ll 1$. The first change in the flow topology occurs as increasing $R e_{\mathrm{a}}$ passes $R e_{\mathrm{a}}=934: r_{\mathrm{s}}$, becomes zero, i.e., layer CR6 vanishes, and region CR2 (figure 21(b)) extends from the axis to the sidewall for $R e_{\mathrm{a}}>934$ (figure 26(b)).

\subsection{Topological flow metamorphoses in the $\alpha=120^{\circ}$ cone as Re increases}

Figure 27 depicts the streamline patterns at $R e=1000$ (a) and 2000 (b) for $H_{\mathrm{w}}=0.65$ and $\alpha=120^{\circ}$. At $R e=1000$ (figure 27(a)), the flow topology is the same as in the creeping flow 
Table 1. As water level $H_{\mathrm{w}}$ increases, VB develops in water (air) at $H_{\mathrm{w}}=H_{\mathrm{ww}}\left(H_{\mathrm{wa}}\right)$. $N$ is a number of topological transformations, as $H_{\mathrm{w}}$ varies from 0 to 1 , and $\alpha$ is the bottom-sidewall angle.

\begin{tabular}{llll}
\hline$\alpha$ & $H_{\mathrm{ww}}$ & $H_{\mathrm{wa}}$ & $N$ \\
\hline $60^{\circ}$ & 0.81 & 0.85 & 18 \\
$90^{\circ}$ & 0.64 & 0.705 & 10 \\
$120^{\circ}$ & 0.563 & 0.648 & 6 \\
\hline
\end{tabular}

(figure 13(c)). The quantitative difference is that the radial extent, $r_{\mathrm{S}}$, of layer CR6 becomes smaller at $R e_{\mathrm{a}}=67$ than that for $R e_{\mathrm{a}} \ll 1$. The first change in the flow topology occurs as increasing $R e_{\mathrm{a}}$ passes $R e_{\mathrm{a}}=130: \mathrm{r}_{\mathrm{s}}$, becomes zero, i.e., layer CR6 vanishes, and region CR2 (figure 13(c)) extends from the axis to the sidewall for $\operatorname{Re}_{\mathrm{a}}>130$ (figure 27(b)).

Thus, the flow topology, described for the creeping flows, remains unchanged until $R e_{\mathrm{a}}$ exceed 130 .

\section{Concluding remarks}

This paper describes effects of a conical sidewall on an air-water motion, driven by the top rotating disk, in a bioreactor and reveals a variety of flow patterns as the water level, $H_{\mathrm{w}}$, grows from 0 to 1 . In contrast to the single-fluid flow (Escudier et al 2007), VB and other topological metamorphoses occur in this two-fluid flow even if it is very slow.

\subsection{Merging of the interface and Moffatt eddies}

First, our study revealed a new topological transformation in the shallow-water flow (section 4). Similar to the corner eddies (Moffatt 1964), unbounded sets of eddies develop near the sidewall above and below the interface (Shtern 2014). As $H_{\mathrm{w}}$ decreases, the outmost Moffat and lower-interface eddies merge into a peripheral ring cell (section 4.1). The corresponding $H_{\mathrm{w}}$ value diminishes (grows) as bottom-sidewall angle $\alpha$ increases (decreases) as shown in section 4.2 (section 4.3). The clockwise water circulation at the periphery is driven by the outmost air eddy while the anticlockwise circulation of water near the axis is driven by the bulk air flow (figure 6(a)).

\subsection{Effect of the cone angle on $V B$}

Second, we found the effects of $\alpha$ on VB occurrence in the water and air flows (at $\alpha=120^{\circ}$ in section 5 and $\alpha=60^{\circ}$ in section 6) summarized in table 1 . There $H_{\mathrm{ww}}$ is the $H_{\mathrm{w}}$ value at which VB occurs in the water flow, $H_{\mathrm{wa}}$ is the $H_{\mathrm{w}}$ value at which VB occurs in the air flow, and $N$ is a number of topological changes in the flow pattern as $H_{\mathrm{w}}$ varies from 0 to 1 . The revealed multiple changes are due to competing effects of AMF (the air meridional flow) and swirl on the water motion and due to the water-flow feedback to the air motion as explained below.

\subsection{Physical reasons for the changes in flow topology}

The rotating top disk induces a centrifugal force which pushes air to the periphery near the disk and thus develops a clockwise meridional circulation, e.g. as shown in figure 10(b). Therefore, air converges toward the axis near the interface and drives the bulk anticlockwise 
circulation of water. In addition, the air circulation induces a set of the Moffatt eddies near the interface-sidewall intersection which generate slave eddies in water. This scenario works for small $H_{\mathrm{w}}$ where the direct effect of centrifugal force in water is negligible compared with the air meridional flow effect, because swirl drastically decays from the top to the bottom.

As $H_{\mathrm{w}}$ increases, the effect of swirl becomes first dominating near the axis-bottom intersection (Herrada and Shtern 2014) and causes the emergence of meridional countercirculation VBB in water. With increasing $H_{\mathrm{w}}$, the clockwise (anticlockwise) water circulation expands (shrinks). After VBB reaches the interface, it reverses the radial velocity of adjacent air developing a TCL (thin circulation layer) attached to the interface. This TCL is narrow due to the small air-to-water density ratio.

As $H_{\mathrm{w}}$ approaches 1 , the effect of swirling water overcomes the direct effect of swirling air, resulting in that the TCL expands up to the sidewall and up to the rotating disk while the clockwise circulation in air shrinks to the sidewall-lid intersection. Interaction of these bulk air and water circulations with the Moffatt and interface eddies enriches the variety of topological transformations. This bifurcation diversity of the creeping air-water flow also remain valid for finite values of the Reynolds number up to $R e_{\mathrm{a}}$ around 100 , depending on $\alpha$ (section 7).

The multi-cell flow patterns can be beneficial for bioreactors, because they provide fine and soft mixing, which is necessary for the tissue growth, and do not destroy the tissue culture since shear stresses are small in a slow motion. Thus, the obtained results are of both fundamental and technological interest.

\section{Acknowledgments}

Partial support from the Ministry of Science and Education and Junta de Andalucía (Spain) through grants nos. DPI2010-21103 and P08-TEP-04128, respectively, is gratefully acknowledged. Adnan Balci is supported by the Turkish Military Academy.

\section{References}

Benjamin T B 1962 Theory of vortex breakdown phenomenon J. Fluid Mech. 14 593-629

Blake J 1979 On the generation of viscous toroidal eddies in a cylinder J. Fluid Mech. 95 209-22

Brøns M 1994 Topological fluid dynamics of interfacial flows Phys. Fluids 6 2730-7

Brøns M 2007 Streamline topology: patterns in fluid flows and their bifurcations Adv. Appl. Mech. 41 $1-42$

Escudier M P 1988 Vortex breakdown: observations and explanations Prog. Aerosp. Sci. 25189

Escudier M P, O'Leary J and Poole R J 2007 Flow produced in a conical container by a rotating endwall Int. J. Heat Fluid Flow 28 1418-28

Gürcan F, Gaskell P H, Savage M D and Wilson M C T 2003 Eddy genesis and transformations of Stokes Flow in a double-lid driven cavity Proc. Inst. Mech. Eng. C: J. Mech. Eng. Sci. 217 353-64

Hall O, Hills C P and Gilbert A D 2007 Slow flow between concentric cones Q. J. Mech. Appl. Math. 60 27-48

Herrada M A and Shtern V N 2014 Patterns of a creeping water-spout flow J. Fluid Mech. 744 65-88

Herrada M A, Shtern V N and López-Herrera J M 2013 Vortex breakdown in a water-spout flow Phys. Fluids 25093604

Herrada M A, Shtern V N and Torregrosa M A 2015 The instability nature of the Vogel-Escudier flow J. Fluid Mech. 766 590-610

Hills C P 2001 Eddies induced in cylindrical containers by a rotating end wall Phys. Fluids 13 2279-86

Leibovich S 1978 The structure of vortex breakdown Annu. Rev. Fluid Mech. 10 221-46 
Liow K Y S, Tan B T, Thouas G and Thompson M C 2009 CFD modeling of the steady-state momentum and oxygen transport in a bioreactor that is driven by a rotating disk Mod. Phys. Lett. B 23 121-7

Lo Jacono D, Nazarinia M and Brøns M 2009 Experimental vortex breakdown topology in a cylinder with a free surface Phys. Fluids 21111704

Lopez J M 1990 Axisymmetric vortex breakdown: I. Confined swirling flows J. Fluid Mech. 221 $533-52$

Moffatt H K 1964 Viscous and resistive eddies near a sharp corner J. Fluid Mech. 18 1-18

Mullin T, Kobine J J, S. J. Tavener S J and Cliffe K A 2000 On the creation of stagnation points near a straight and sloped walls Phys. Fluids 12 425-32

Ramazanov Y A, Kislykh V I, Kosyuk I P, Bakuleva N V and Shchurikhina V V 2007 Industrial production of vaccines using embryonic cells in gas-vortex gradient-less bioreactors New Aspects of Biotechnology and Medicine ed A M Egorov pp 87-91 (New York: Nova Science)

Schultz S, Gorbach G and Piesche M 2009 Modeling fluid behavior and droplet interactions during liquid-liquid separation in hydrocyclones Chem. Eng. Sci. 643935

Secchiaroli A, Ricci R, Montelpare S and D'Alessandro V 2009 Numerical simulation of turbulent flow in a Ranque-Hilsch vortex-tube Int. J. Heat Mass Transfer 525496

Shankar P N and Deshpande M D 2000 Fluid mechanics in the driven cavity Annu. Rev. Fluid Mech. 32 93-136

Shtern V 2012a Counterflows (Cambridge: Cambridge University Press)

Shtern V 2012b A flow in the depth of infinite annular cylindrical cavity J. Fluid Mech. 711 667-80

Shtern V 2014 Moffatt eddies at an interface Theor. Comput. Fluid Dyn. 28 651-6

Shtern V and Hussain F 1999 Collapse, symmetry breaking, and hysteresis in swirling flows Annu. Rev. Fluid Mech. 31 537-66

Valentine D T and Jahnke C C 1994 Flow induced in a cylinder with both endwalls rotating Phys. Fluids 6 2702-10

Wakiya S 1976 Axisymmetric flow of a viscous fluid near the vertex of a body J. Fluid Mech. 78 737-47

Wilson M C T, Gaskell P H and Savage M D 2005 Nested separatrices in simple shear flows: the effect of localized disturbances on stagnation lines Phys. Fluids 17093601 